\title{
Two-Center Black Holes Duality-Invariants for stu Model and its lower-rank Descendants
}

\author{
Sergio Ferrara ${ }^{a, b, c}$, Alessio Marrani ${ }^{a}$, Emanuele Orazi ${ }^{a}$ \\ Raymond Stora $^{a, d}$ and Armen Yeranyan ${ }^{b, e}$ \\ a Physics Department, Theory Unit, CERN, \\ CH -1211, Geneva 23, Switzerland; \\ ${ }^{b}$ INFN - Laboratori Nazionali di Frascati, \\ Via Enrico Fermi 40, I-00044 Frascati, Italy \\ ayeran@lnf. infn.it \\ ${ }^{c}$ Department of Physics and Astronomy, \\ University of California, Los Angeles, CA 90095-1547,USA \\ ${ }^{d}$ LAPTH, Université de Savoie, CNRS \\ Annecy-le-Vieux, France \\ e Department of Physics, Yerevan State University \\ Alex Manoogian St. 1, Yerevan, 0025, Armenia
}

\begin{abstract}
We classify 2-center extremal black hole charge configurations through duality-invariant homogeneous polynomials, which are the generalization of the unique invariant quartic polynomial for single-center black holes based on homogeneous symmetric cubic special Kähler geometries.

A crucial role is played by an horizontal $S L(p, \mathbb{R})$ symmetry group, which classifies invariants for $p$-center black holes. For $p=2$, a (spin 2) quintet of quartic invariants emerge. We provide the minimal set of independent invariants for the rank-3 $\mathcal{N}=2, d=4$ stu model, and for its lower-rank descendants, namely the rank- $2 s t^{2}$ and rank-1 $t^{3}$ models; these models respectively exhibit seven, six and five independent invariants.

We also derive the polynomial relations among these and other duality invariants. In particular, the symplectic product of two charge vectors is not independent from the quartic quintet in the $t^{3}$ model, but rather it satisfies a degree-16 relation, corresponding to a quartic equation for the square of the symplectic product itself.
\end{abstract}




\section{Contents}

1 Introduction $\quad 1$

2 The $s t u$ Model

3 2-Center $G_{4}$-Invariants and The T-tensor Formalism $\quad 7$

4 The Role of the Horizontal Symmetry $S L_{h}(2, \mathbb{R}) \quad[8$

5 Independent Invariants, Constraints and their $s t^{2}$ and $t^{3}$ Descendants $\quad 10$

6 The $s t^{2}$ Model 11

7 The $t^{3}$ Model $\quad 13$

8 Extension to $S O_{h}^{v}(2,2)$ Symmetry and the Gramian Matrix

9 Generalization to $\mathcal{N}=2$ Jordan Symmetric Sequence and $\mathcal{N}=4$ Theory

A Derivation of the Hierarchy of Constraints for $s t u, s t^{2}$ and $t^{3}$ Models

B Constrained Calabi-Vesentini Basis for $t^{3}$ Model 23

C A Complete Basis for $S O(n, \mathbb{C})$-invariant Polynomials $\quad 26$

\section{Introduction}

Multi-center black holes (BHs) are a natural extension of single-center BHs, and they play an important role in the dynamics of quantum theories of gravity, such as superstrings and $M$-theory.

In fact, interesting multi-center solutions have been found for BPS BHs in $d=4$ theories with $\mathcal{N}=2$ supersymmetry, in which the Attractor Mechanism [1, 2] for static, spherically symmetric, asymptotically flat, extremal dyonic BHs is generalised by the so-called split attractor flow [3, 4, 5. This name comes from the existence, for 2-center solutions, of a co-dimension one region (named marginal stability wall) in the scalar manifold, where in fact a stable 2-center $\mathrm{BH}$ configuration may decay into two single-center constituents, whose scalar flows then separately evolve according to the corresponding attractor dynamics.

The study of these phenomena has recently progressed in many directions. By combining properties of $\mathcal{N}=2$ supergravity and superstring theory, a number of interesting phenomena, such as split flow tree, entropy enigma, bound state recombination walls, and microstate counting have been investigated (see e.g. 6]-[17]; as examples of earlier studies, see e.g. [18]).

In the supergravity approximation, the detailed study of the split attractor flow is made possible, in the limit of large (continuous) charges, by the powerful restrictions imposed by electric-magnetic duality ( $U$-duality 1 ).

An important ingredient in the study of attractor solutions in supergravity is the concept of duality charge orbits, and of the duality invariants associated to them. In the past, a number of studies has

\footnotetext{
${ }^{1}$ Here $U$-duality is referred to as the "continuous" limit (valid for large values of the charges) of the non-perturbative string theory symmetries introduced by Hull and Townsend in 19.
} 
led to a rather complete classification of charge orbits for single-center extremal BHs, and of their supersymmetry-preserving properties characterising the corresponding $\mathrm{BH}$ background [20]- 28].

After [2, it is known that in generic $\mathcal{N}=2$ theories all scalar fields (belonging to Abelian vector multiplets) are stabilized in terms of the charges in the near-horizon extremal BH geometry corresponding to a $\left(\frac{1}{2}-\right)$ BPS attractor configuration. On the other hand, for $\mathcal{N}=2$ non-BPS attractors, as well as for both BPS and non-BPS attractors in $\mathcal{N}>2$-extended theories, not all scalar fields are stabilized at the $\mathrm{BH}$ event horizon [29], and "moduli spaces" of attractor solutions exist [30].

In $d=4$ supergravity theories, the fluxes of the two-form Abelian field-strengths and their duals fit into the relevant (symplectic) irrepr. $\mathbf{R}$ of the $U$-duality group $G_{4}$. When considering a(n extremal) 1-center black 0-brane (BH) background, such fluxes are referred to as electric and magnetic black hole charges. The irrepr. charge space $\mathbf{R}$ exhibits a stratification in terms of disjoint orbits, each of them supporting a distinct class of 1-center BH solutions [20]-28]. Within theories with symmetric coset scalar manifolds $\frac{G_{4}}{H_{4}}$ (where $H_{4}$ is the maximal symmetric subgroup of $G_{4}$ ), a unique duality-invariant polynomial of the charge irrepr. $\mathbf{R}$ of $G_{4}$ (in which the charges of a 1-center BH sit) exists.

This is a quadratic polynomial $\mathcal{I}_{2}$ in $\mathcal{N}=2$ symmetric special Kähler (SK) geometries with vanishing $C$-tensor (minimal coupling sequence [31]). The same is also true for the general $\mathcal{N}=3$ theory [32] and for "pure" $\mathcal{N}=4$ supergravity [33] (the so-called axion-dilaton model, whose truncation down to $\mathcal{N}=2$ gives rise to the $\mathbb{C P}^{1}$ model - first element of the minimal coupling sequence - in a non-manifestly $U(1,1)$-covariant symplectic basis).

On the other hand, symmetric $d$-special Kähler geometries (for a comprehensive treatment, see e.g. [34]), based on degree-3 Euclidean Jordan algebras [35, 36], have a unique duality-invariant polynomial $\mathcal{I}_{4}$ which is quartic in charges. Some of these theories correspond to certain classical limits of moduli spaces of Calabi-Yau internal manifolds in superstring compactifications [12]. In particular, the simplest $d$-SK geometry, namely the symmetric $t^{3}$ model (see Sec. 7, and Refs. therein), pertains to the volume modulus in the large volume limit of compactifications of Type II superstrings on Calabi-Yau threefolds.

For $p$-center (extremal) BHs, the study of charge orbits and duality invariants is not known yet. The unique exception is given by the above mentioned minimal coupling $\mathbb{C P}^{n}$ sequence; indeed, the 2-center dynamics, marginal stability and the properties of the related split attractor flows have been recently shown to depend on four $U$-duality invariants in [37] (in the same paper, a generalisation to $\mathrm{p}(\geqslant 3)$-center solutions was indicated, as well).

The present investigation is devoted to the study of duality charge orbits supporting 2-center extremal BHs in $\mathcal{N}=2$ symmetric $d$-SK geometry based on the so-called Jordan symmetric infinite sequence [36]

$$
\mathcal{N}=2: \frac{S L(2, \mathbb{R})}{U(1)} \times \frac{S O(2, n)}{S O(2) \times S O(n)}\left(\mathbb{R} \oplus \boldsymbol{\Gamma}_{1, n-1}\right),
$$

where the round brackets in the right-hand side denote the corresponding reducible degree-3 Euclidean Jordan algebras [35, 36] (see also e.g. [38, and Refs. therein). In particular, we will focus on the symmetric minimal rank-3 stu model [40, 41] (which is a sub-sector of all symmetric $d$-SK geometries) and its lower-rank descendants, namely the $s t^{2}$ and $t^{3}$ models.

In general, in presence of a $p$ center (extremal) $\mathrm{BH}$ solution $(p \in \mathbb{N})$, the number $I_{p}$ of independent $G_{4}$-invariant polynomials built out with $p$ distinct copies of the charge irrepr. $\mathbf{R}$ of $G_{4}$ is given by the formula

$$
p \operatorname{dim}_{\mathbb{R}} \mathbf{R}=\operatorname{dim}_{\mathbb{R}} \mathcal{O}_{p}+I_{p}
$$

where

$$
\mathcal{O}_{p} \equiv \frac{G_{4}}{\mathcal{H}_{4, p}}
$$

is the relevant $p$-center charge orbit, spanned by a vector of fluxes of real dimension $p \operatorname{dim}_{\mathbb{R}} \mathbf{R}$. In general, the counting of $I_{p}$ given by the formulæ (1.2)-(1.3) depends only on the compact form of the 
symmetry groups involved, and thus it is not affected by the supersymmetry properties exhibited by the corresponding $p$-center BH background.

For example, in the case of BPS $p$-center extremal BHs in $\mathcal{N}=2$ minimally coupled supergravity [31], one obtains $(p \leqslant n+1$; see Sec. 4.2.1 of [37])

$$
\left.\begin{array}{c}
p \operatorname{dim}_{\mathbb{R}} \mathbf{R}=2(n+1) p ; \\
\mathcal{O}_{B P S, p}=\frac{U(1, n)}{U(n+1-p)}
\end{array}\right\} \Rightarrow I_{p}=p^{2} .
$$

A new phenomenon occurring when $p>1$ is the fact that the various $G_{4}$-invariant polynomials arrange into irreprs. (multiplets) of an "horizontal" symmetry group, encoding the combinatoric structure of the $p$-center solutions of the theory. In the $\mathcal{N}=2$ minimally coupled theory, such an "horizontal" group is given by $U_{h}(p)$ [37] (the subscript " $h$ " stands for "horizontal" throughout). On the other hand, for the cubic models considered in the present paper it is $22 L_{h}(p, \mathbb{R})$ (see Sec. 4).

For all $\mathcal{N}=2$ theories, $\operatorname{dim}_{\mathbb{R}} \mathbf{R}=2 n_{V}+2$, where $n_{V}$ is the number of Abelian vector multiplets coupled to the gravity multiplets. Thus, at least for $\mathcal{N}=2$ symmetric coset vector multiplets' scalar manifolds, the dimension of a "large" charge orbit $\mathcal{O}_{p=1}$ reads

$$
\operatorname{dim}_{\mathbb{R}} \mathcal{O}_{p=1}=2 n_{V}+1=\operatorname{dim}_{\mathbb{R}}\left(\frac{G_{4}}{H_{4,0}}\right)=\operatorname{dim}_{\mathbb{R}} \mathbf{R}-1
$$

where $H_{4}=H_{4,0} \times U(1)$ is the maximal compact subgroup of $G_{4}$, as well as the stabilizer of the scalar manifold itself. Thus, the application of general relation (1.2) to the $\mathcal{N}=21$-center case (1.5) (which can be traced back to the very structure of SK geometry [20, 23]) yields to the well known result

$$
I_{p=1}=\operatorname{dim}_{\mathbb{R}} \mathbf{R}-\operatorname{dim}_{\mathbb{R}} \mathcal{O}_{p=1}=1,
$$

and the "large" nature of $\mathcal{O}_{p=1}$ means that it supports a non-vanishing value of the unique $G_{4}$-invariant.

As mentioned above, we will focus on the $s t u, s t^{2}$ and $t^{3} d$-SK geometries, respectively corresponding to the rank-3, rank-2 and rank-1 symmetric cosets (see the treatment of Secs. 2, 6] and 7 for more detail, and Refs. Therein):

$$
\left[\frac{S L(2, \mathbb{R})}{\underset{\mathcal{F}=s t u}{U(1)}}\right]^{3},\left[\frac{S L(2, \mathbb{R})}{\underset{\mathcal{F}=s t^{2}}{U(1)}}\right]^{2}, \frac{S L(2, \mathbb{R})}{\underset{\mathcal{F}=t^{3}}{U(1)}}
$$

The charge irrepr. $\mathbf{R}$ respectively is the $(\mathbf{2}, \mathbf{2}, \mathbf{2})$ ( $\left.\operatorname{spin} s=\left(\frac{1}{2}, \frac{1}{2}, \frac{1}{2}\right)\right)$ of $[S L(2, \mathbb{R})]^{3}$, the $(\mathbf{3}, \mathbf{2})$ (spin $\left.s=\left(1, \frac{1}{2}\right)\right)$ of $[S L(2, \mathbb{R})]^{2}$, and the $(4)\left(\operatorname{spin} s=\frac{3}{2}\right)$ of $[S L(2, \mathbb{R})]$ (see also the discussion in Sec. 5 of [42]). For these models, the generic ("large") $p=2$-center charge orbit $\mathcal{O}_{p=2}$ has no continuous stabilizer, so it just coincides with $G_{4}$ itself. Thus, the application of the general formula (1.2) with $p=2$ in the theories under consideration yields that

$$
I_{p=2}=\underset{s t u}{7}, \underset{s t^{2}}{6}, \quad \underset{t^{3}}{5} .
$$

As discussed in Sec. 3 within the (manifestly $G_{4}$-covariant) so-called Calabi-Vesentin 3 basis [43, 44, 41, a remarkable property of the $s t u$ and $s t^{2}$ models is that $G_{4}$ is reducible (namely, factorised: $G_{4}=[S L(2, \mathbb{R})]^{3}$ for $s t u$, and $G_{4}=[S L(2, \mathbb{R})]^{2}$ for $s t^{2}$ ). This generally allows for the existence of more independent $G_{4}$-invariant polynomials with respect to symmetric theories with irreducible $G_{4}$ (such as the $\mathcal{N}=2$ "magic" models [35, 36]). Actually, both the whole $\mathcal{N}=2$ Jordan symmetric

\footnotetext{
${ }^{2}$ Actually, in these cases the horizontal symmetry group is $G L(p, \mathbb{R})$, where the additional scale symmetry with respect to $S L(p, \mathbb{R})$ is encoded by the homogeneity of the $G_{4}$-invariant polynomials in charges.

${ }^{3}$ The Calabi-Vesentini basis for charges and holomorphic sections is discussed in App. B
} 
sequence (1.1) (whose the $s t u$ and $s t^{2}$ models are the $n=2$ and $n=1$ element, respectively) and the sequence pertaining to $\mathcal{N}=4$ supergravity (see Eq. (9.1) further below) have factorised scalar manifolds, and the aforementioned property (as well as the possibility to perform a Calabi-Vesentini manifestly $G_{4}$-covariant treatment) extends to these two infinite sequences. It is here worth pointing out that, for symmetric $d$-SK geometries, the reducible (irreducible) nature of $G_{4}$ is ultimately due to the reducibility (irreducibility) of the underlying rank-3 Euclidean Jordan algebra (for the reducible cases, see Eqs. (1.1) and (9.1)).

For the stu model, in Sec. 2 we will show that there is a basis of seven independent $[S L(2, \mathbb{R})]^{3}$ invariant homogeneous polynomials, six of them are quartic and the one is quadratic in charges. Within the notation specified in Sec. 4, these polynomial invariants arrange as follows:

$$
\mathcal{W} \equiv\left\langle\mathcal{Q}_{1}, \mathcal{Q}_{2}\right\rangle \text { quadratic in charges (see Eq. (4.11)) }
$$

$(\mathfrak{I}, \mathcal{X})$ quartic in charges (see Eq. (4.5)),

where $\mathfrak{I}$ is a quintet of five invariants, and $\left\langle\mathcal{Q}_{1}, \mathcal{Q}_{2}\right\rangle$ denotes the symplectic product of the charge vectors pertaining to the two centers.

For the $s t^{2}$ and $t^{3}$ models, in which the number of independent $G_{4}$-invariants is smaller, we will exhibit polynomial constraints, manifestly invariant under the aforementioned "horizontal" symmetry $S L_{h}(2, \mathbb{R})$, which relate such invariants. We anticipate that both $\mathcal{W}$ and $\mathcal{X}$ of Eq. (1.9) are singlets under $S L_{h}(2, \mathbb{R})$, whereas $\mathfrak{I}$ sit in an irrepr. 5 (spin $s=2$ ) of $S L_{h}(2, \mathbb{R})$ itself (see(4.5)). In the $s t u$ model, the relevant polynomial constraint has the (order-12 in charges) structure (see Eq. (5.6) for explicit form):

$$
\mathcal{P}_{12}\left(\mathbf{I}_{6}, \mathcal{W}, \mathcal{X}, \operatorname{Tr}\left(\mathfrak{I}^{2}\right), \operatorname{Tr}\left(\mathfrak{I}^{3}\right)\right)=0
$$

which always allows one e.g. to eliminate $\operatorname{Tr}\left(\mathfrak{I}^{3}\right)$ in terms of $\mathbf{I}_{6}$, and vice versa. As detailed in App. A. the further reduction to the $s t^{2}$ model gives rise to a polynomial of order-16 in charges structure (see Eqs. (6.10) and (6.12) for explicit form):

$$
\mathcal{P}_{16}\left(\mathcal{W}, \mathcal{X}, \operatorname{Tr}\left(\mathfrak{I}^{2}\right), \operatorname{Tr}\left(\mathfrak{I}^{3}\right)\right)=0,
$$

and it can be regarded a fourth order algebraic equation for $\mathcal{W}^{2}$. In the $t^{3}$ model, a relation of type (1.11) (with $\mathcal{P}_{16}$ given by (6.12) ) also holds, together with the further constraint $\mathcal{X}=0$ (see Eq. (7.16) ), of order 4 in charges. Note that the quintet $\mathfrak{I}$ enters Eqs. (1.10) and (1.11) only through the $S L_{h}(2, \mathbb{R})$-invariant expressions $\operatorname{Tr}\left(\mathfrak{I}^{2}\right)$ and $\operatorname{Tr}\left(\mathfrak{I}^{3}\right)$, respectively given by Eqs. (4.9) and (4.10).

Alternatively, by using (1.10), one can replace $\operatorname{Tr}\left(\mathfrak{I}^{3}\right)$ with $\mathbf{I}_{6}$ (defined in (3.16)) as generator of a complete lowest-degree basis (8.2) of manifestly $S L_{h}(2, \mathbb{R})$-invariant polynomials. Thus, Eq. (1.11) gets replaced by the degree- 8 constraint (6.11), which is nothing but the vanishing of the determinant of a suitably defined Gramian matrix $\mathbf{G}$ (defined by (8.4)-(8.5)).

The paper is organised as follows.

In Sec. 2 we recall the $s t u$ model and its properties (in the "special coordinates" symplectic frame).

In Sec. 3 we introduce a general formalism for the construction and analysis of polynomial $G_{4^{-}}$ invariants in all cases in which $G_{4}$ is factorised. This formalism is worked out in the Calabi-Vesentini basis [44, and it is based on the so-called $\mathbb{T}$-tensor; we also briefly outline the relation between the $\mathbb{T}$-tensor and the corresponding counterpart for irreducible cubic geometries. In particular, this formalism applies to $s t u$ and $s t^{2}$ since they are, as mentioned above, the $n=2$ and $n=1$ element of the $\mathcal{N}=2$ Jordan symmetric sequence (1.1), respectively. The application to the rank-1 irreducible $t^{3}$ model deserves a separate treatment, given in App. B.

Sec. 4 analyzes the crucial role played by the "horizontal" symmetry $S L_{h}(2, \mathbb{R})$ (generalisable to $S L_{h}(p, \mathbb{R})$ for $p \geqslant 3$ centers) in classifying the polynomial $G_{4}$-invariants and in determining the structure of the polynomial constraints relating them. In particular, for each order of homogeneity 
in charges, the various $G_{4}$-invariants arrange into irreprs. (multiplet) of the "horizontal" symmetry itself.

In Sec. 5 the issue of independence (primitivity) of the $G_{4}$-invariant in the models under consideration is addressed. Besides the explicit computation based on the analysis of the rank of a suitably defined Jacobian matrix, also the general counting argument based on formula (1.2) is given. A polynomial constraint of degree 12 in charges, involving also the unique $G_{4}$-invariant polynomial of order six in charges (singlet under $S L_{h}(2, \mathbb{R})$ ) is given (and derived in detail in App. A).

Then, in Secs. 6 and 7 the reduction of the $s t u$ to $s t^{2}$ respectively $t^{3}$ model is performed, and in App. A the corresponding hierarchy of manifestly $S L_{h}(2, \mathbb{R})$-invariant polynomial constraints (consistent with the result (1.8) ) is derived.

In Sec. 8 we develop further the analysis of invariant polynomials, by combining the "horizontal" symmetry $S L_{h}(2, \mathbb{R})$ with the "vertical" symmetry $S L_{v}(2, \mathbb{R})$. This latter, for the models treated in the present paper, is part of the $d=4 U$-duality group $G_{4}$. Then, we use the characteristic equation of the Gramian matrix $\mathbf{G}$ to exploit a manifestly $\left[S L_{h}(2, \mathbb{R}) \times S L(2, \mathbb{R})\right]$-invariant formalism, actually holding for both the infinite reducible sequences (1.1) and (9.1) of symmetric scalar manifolds.

Finally, in Sec. 9 the extension of the previous analysis to generic elements of the $\mathcal{N}=2$ Jordan symmetric and $\mathcal{N}=4$ reducible infinite sequences is discussed; for the $\mathcal{N}=2$ sequence with $n \geqslant 3$ and for the whole $\mathcal{N}=4$ sequence $(n \geqslant 0)$, the treatment is analogous, and the results identical, to the case of the stu model considered in Sec. 2,

Three Appendices conclude the paper. In App. $\mathrm{A}$ we give details on the derivation of the relevant polynomial constraints in $s t u, s t^{2}$ and $t^{3}$ models. App. B discusses the relation between the usual "special coordinates" symplectic basis (used in $D$-brane description) and the Calabi-Vesentini basis. App. Clpresents a complete basis for the $S O(n, \mathbb{C})$-invariant polynomials, a rigorous result mentioned in Sec. 8 ,

We should point out that, although we perform an analysis for BPS ("large") multi-center extremal BHs, the extension to non-BPS "large" as well as to "small" BHs is straightforward. Strictly speaking, it is worth recalling that, at the best of our current understanding (see e.g. [39]), the marginal decay and split attractor flow can be generalised to $\mathcal{N}=2$ non-BPS cases only with $\mathcal{I}_{4}>0$ (namely, the $Z_{H}=0$ attractors). Anyhow, the analysis of $p$-center charge orbits can be carried out for all cases (see the comments below Eq. (1.3)).

\section{The stu Model}

We start and consider the so-called $\mathcal{N}=2, d=4$ stu model [40, 41]. In the "special coordinates" basis (see e.g. [45] and Refs. therein), this model is defined by the prepotential

$$
F(X) \equiv \frac{X^{1} X^{2} X^{3}}{X^{0}}=\frac{1}{3 !} d_{i j k} \frac{X^{i} X^{j} X^{k}}{X^{0}} \Leftrightarrow d_{123}=1,
$$

thus implying

$$
\begin{aligned}
F_{0} & =\frac{\partial F}{\partial X^{0}}=-X^{0} \mathcal{F} ; \quad F_{1}=\frac{\partial F}{\partial X^{1}}=X^{0} \mathcal{F}_{1} ; F_{2}=\frac{\partial F}{\partial X^{2}}=X^{0} \mathcal{F}_{2} ; \quad F_{3}=\frac{\partial F}{\partial X^{3}}=X^{0} \mathcal{F}_{3} ; \\
\mathcal{F} & \equiv s t u ; \mathcal{F}_{1} \equiv t u=\frac{\partial \mathcal{F}}{\partial s} ; \mathcal{F}_{2} \equiv s u=\frac{\partial \mathcal{F}}{\partial t} ; \mathcal{F}_{3} \equiv s t=\frac{\partial \mathcal{F}}{\partial u}
\end{aligned}
$$

where

$$
s \equiv \frac{X^{1}}{X^{0}}, t \equiv \frac{X^{2}}{X^{0}}, u \equiv \frac{X^{3}}{X^{0}}
$$


are the projective coordinates. Through the definition (2.4), the $S p(8, \mathbb{R})$-vector of holomorphic symplectic sections can thus be written as follows:

$$
\mathbf{V} \equiv\left(\begin{array}{c}
X^{0} \\
X^{1} \\
X^{2} \\
X^{3} \\
F_{0} \\
F_{1} \\
F_{2} \\
F_{3}
\end{array}\right)=\left(\begin{array}{c}
1 \\
s \\
t \\
u \\
-\frac{1}{2} s t^{2} \\
t u \\
s u \\
s t
\end{array}\right) X^{0}=\left(\begin{array}{c}
1 \\
s \\
t \\
u \\
-\mathcal{F} \\
\mathcal{F}_{1} \\
\mathcal{F}_{2} \\
\mathcal{F}_{3}
\end{array}\right) X^{0}
$$

Here we will not report a detailed treatment of the stu model (we address the reader e.g. to [40, 41, 42, 46, 47]), we will just confine ourselves to some basics, useful for the developments given below.

The stu model is based on the rank-3 completely factorised symmetric coset

$$
\frac{G_{4}}{H_{4}}=\frac{S L(2, \mathbb{R})}{U(1)} \times \frac{S O(2,2)}{S O(2) \times S O(2)} \sim\left[\frac{S L(2, \mathbb{R})}{U(1)}\right]^{3},
$$

where $G_{4}=[S L(2, \mathbb{R})]^{3}$ is the $d=4 U$-duality group, and $H_{4}=[U(1)]^{3}$ its maximal compact subgroup (mcs). This coset is the second element $(n=2)$ of the aforementioned $\mathcal{N}=2, d=4$ Jordan symmetric sequence (see e.g. [48, 34], and Refs. therein).

This model admits all classes of extremal BH attractors [1, 2], namely $\frac{1}{2}$-BPS, non-BPS $Z_{H} \neq 0$ and non-BPS $Z_{H}=0$ ones. The BPS solutions were known after [41, 49, whereas the explicit expression of the non-BPS $Z_{H}=0$ attractors have been obtained in [42. The non-BPS $Z_{H} \neq 0$ attractor solutions were obtained in full generality in [46] (see also Refs. therein, as well as [47]).

By introducing the $S p(8, \mathbb{R})$-vector of magnetic and electric charges (the naught index pertains to the graviphoton throughout)

$$
\mathcal{Q} \equiv\left(p^{0}, p^{1}, p^{2}, p^{3}, q_{0}, q_{1}, q_{2}, q_{3}\right)^{T},
$$

in the "special coordinate basis" the unique polynomial invariant (homogeneous and quartic in the charges) of the $(\mathbf{2}, \mathbf{2}, \mathbf{2})$ (namely spin $\left.s=\left(\frac{1}{2}, \frac{1}{2}, \frac{1}{2}\right)\right)$ irrepr. of $G_{4}=[S L(2, \mathbb{R})]^{3}$ reads

$$
\begin{aligned}
\mathcal{I}_{4}(\mathcal{Q}) \equiv & -\left(p^{0}\right)^{2} q_{0}^{2}-\left(p^{1}\right)^{2} q_{1}^{2}-\left(p^{2}\right)^{2} q_{2}^{2}-\left(p^{3}\right)^{2} q_{3}^{2} \\
& -2 p^{0} q_{0} p^{1} q_{1}-2 p^{0} q_{0} p^{2} q_{2}-2 p^{0} q_{0} p^{3} q_{3}+2 p^{1} q_{1} p^{2} q_{2}+2 p^{1} q_{1} p^{3} q_{3}+2 p^{2} q_{2} p^{3} q_{3} \\
& +4 q_{0} p^{1} p^{2} p^{3}-4 p^{0} q_{1} q_{2} q_{3}=-\operatorname{Det}(\psi),
\end{aligned}
$$

where $\operatorname{Det}(\psi)$ is the so-called Cayley's hyperdeterminant [50]. $\mathcal{I}_{4}>0$ for $\frac{1}{2}$-BPS and non-BPS $Z_{H}=0$, while $\mathcal{I}_{4}<0$ for non-BPS $Z_{H} \neq 0$ attractor solutions, respectively (see Appendix II of [23]). (2.8) can be obtained from the general formula (for symmetric $d$-SK geometries; see [51] for notation and further elucidation)

$$
\mathcal{I}_{4}(\mathcal{Q})=-\left(p^{0} q_{0}+p^{i} q_{i}\right)^{2}+\frac{2}{3} q_{0} d_{i j k} p^{i} p^{j} p^{k}-\frac{2}{3} p^{0} d^{i j k} q_{i} q_{j} q_{k}+d_{i j k} d^{i l m} p^{j} p^{k} q_{l} q_{m},
$$

by specifying $d_{123}=1=d^{123}$, consistently with the non-linear relation (for symmetric $d$-SK geometries [36, 52])

$$
d_{r(p q} d_{i j) k} d^{r k l}=\frac{4}{3} \delta_{(p}^{l} d_{q i j)}
$$

At the level of 1 -center quartic $G_{4}$-invariant polynomials, the progressive reduction "stu $\rightarrow s t^{2} \rightarrow t^{3}$ " procedure has been discussed in Sect. 5 of [42]. 


\section{2-Center $G_{4}$-Invariants and The $\mathbb{T}$-tensor Formalism}

Let us now consider a double-center extremal BH in the stu model, with the charge vectors associated to the two centers respectively reading

$$
\begin{aligned}
& \mathcal{Q}_{1} \equiv\left(p^{0}, p^{1}, p^{2}, p^{3}, q_{0}, q_{1}, q_{2}, q_{3}\right)^{T} ; \\
& \mathcal{Q}_{2} \equiv\left(P^{0}, P^{1}, P^{2}, P^{3}, Q_{0}, Q_{1}, Q_{2}, Q_{3}\right)^{T} .
\end{aligned}
$$

By switching to the so-called Calabi-Vesentini basis [44, 41, in the stu model (and, as we will see below, in the related $s t^{2}$ and $t^{3}$ model, as well) the analysis of the multi-center $U$-invariant polynomials can efficiently be performed by using the following quantity, which we dub "T-tensor" $(\Lambda=0,1,2,3)$ :

$$
\begin{aligned}
\mathbb{T}_{12} & \equiv T_{\Lambda \Sigma}\left(\mathcal{Q}_{1} \mathcal{Q}_{2}\right) \equiv \frac{1}{2}\left(p_{\Lambda} Q_{\Sigma}-q_{\Lambda} P_{\Sigma}+P_{\Lambda} q_{\Sigma}-Q_{\Lambda} p_{\Sigma}\right) ; \\
T_{\Lambda \Sigma}\left(\mathcal{Q}_{1} \mathcal{Q}_{2}\right) & =-T_{\Sigma \Lambda}\left(\mathcal{Q}_{1} \mathcal{Q}_{2}\right)=T_{\Lambda \Sigma}\left(\mathcal{Q}_{2} \mathcal{Q}_{1}\right)=-T_{\Sigma \Lambda}\left(\mathcal{Q}_{2} \mathcal{Q}_{1}\right),
\end{aligned}
$$

where we understand the raising and lowering of $\Lambda$-indices to be done with the metrics $\eta_{\Lambda \Sigma}$ and $\eta^{\Lambda \Sigma}$ of $S O(2,2)$. Note that the 1 -center limit $1 \equiv 2$ of (3.3) consistently yields the antisymmetric rank-2 tensors usually considered in the 1-center analysis (see e.g. [53, 29, 27, 54])

$$
\begin{aligned}
& \mathbb{T}_{1} \equiv T_{\Lambda \Sigma}\left(\mathcal{Q}_{1}^{2}\right) \equiv p_{\Lambda} q_{\Sigma}-q_{\Lambda} p_{\Sigma} \\
& \mathbb{T}_{2} \equiv T_{\Lambda \Sigma}\left(\mathcal{Q}_{2}^{2}\right) \equiv P_{\Lambda} Q_{\Sigma}-Q_{\Lambda} P_{\Sigma}
\end{aligned}
$$

While the charges in the "special coordinates" basis (namely, the ones used in Eq. (2.8)) are manifestly covariant only with respect to the $d=5 U$-duality group $G_{5}=[S O(1,1)]^{2}$, the CalabiVesentini basis is manifestly covariant under the whole $d=4 U$-duality group $S L(2, \mathbb{R}) \times S O(2,2)$ [44]. In the latter basis, by virtue of the factorised nature of the $U$-duality group, the charge vector $\mathcal{Q}$ splits into a magnetic-electric $S L(2, \mathbb{R})$-doublet of $S O(2,2)$ vectors, as follows:

$$
\mathcal{Q}_{\mathbb{A}}=\left(p^{\Lambda}, q_{\Lambda}\right) \equiv\left(Q_{1 \Lambda}, Q_{2 \Lambda}\right) \equiv \mathcal{Q}_{\alpha \Lambda}
$$

where $\alpha=1,2$ is in the fundamental 2 (spin $s=1 / 2)$ irrepr. of $S L(2, \mathbb{R})$, and $\Lambda$ is in the 4 vector irrepr. of $S O(2,2)$. As a consequence, by defining

$$
p^{2} \equiv p^{\Lambda} p^{\Sigma} \eta_{\Lambda \Sigma}, q^{2} \equiv q_{\Lambda} q_{\Sigma} \eta^{\Lambda \Sigma}, p \cdot q \equiv p^{\Lambda} q_{\Lambda},
$$

the unique quartic 1-center $G_{4}$-invariant polynomial (2.8) [55, 40, 56] can be rewritten as follow 4

$$
\mathcal{I}_{4}(\mathcal{Q}) \equiv p^{2} q^{2}-(p \cdot q)^{2}=\frac{1}{2} T_{\Lambda \Sigma}\left(\mathcal{Q}^{2}\right) T_{\Xi \Omega}\left(\mathcal{Q}^{2}\right) \eta^{\Lambda \Xi} \eta^{\Sigma \Omega}=-\frac{1}{2} \operatorname{Tr}\left(\mathbb{T}^{2}\right)
$$

Due to the reducible (factorised) nature of the $d=4 U$-duality group $G_{4}$ in stu and $s t^{2}$ models, the $\mathbb{T}$-tensors $\mathbb{T}_{12}, \mathbb{T}_{1}$ and $\mathbb{T}_{2}$ (defined by $(3.3)$-(3.6) $)$ are the basic structures needed to analyse the $p \geqslant 2$-center $G_{4}$-invariant polynomials. Here below we give the complete analysis of all non-vanishing (a priori) independent invariant polynomials constructed with all possible contractions of two and three $\mathbb{T}$-tensors out of the ones defined by $(3.3)-(3.6)$ :

- two $\mathbb{T}$ 's. For $p=2$ centers, there are six non-vanishing ( $a$ priori) independent invariant polynomials constructed with all possible contractions of two $\mathbb{T}$-tensors out of the ones defined by

\footnotetext{
${ }^{4}$ For reasons of covariance, in Eqs. (3.9), (3.10)-(3.16), (4.3), (4.12), (4.13) and (7.16), "Tr" denotes the $\eta$-trace, namely the trace in which the indices are raised and lowered by the pseudo-Euclidean metric $\eta$.
} 
(3.3)-(3.6), namely (recall definition (3.9)):

$$
\begin{aligned}
\mathbf{I}_{+2}\left(\mathcal{Q}_{1}^{4}\right) & \equiv \mathcal{I}_{4}\left(\mathcal{Q}_{1}\right)=-\frac{1}{2} \operatorname{Tr}\left(\mathbb{T}_{1}^{2}\right)=p^{2} q^{2}-(p \cdot q)^{2} ; \\
\mathbf{I}_{+1}\left(\mathcal{Q}_{1}^{3} \mathcal{Q}_{2}\right) & \equiv-\frac{1}{2} \operatorname{Tr}\left(\mathbb{T}_{1} \mathbb{T}_{12}\right)=\frac{1}{2}\left[p^{2}(q \cdot Q)+q^{2}(p \cdot P)-(p \cdot q)(p \cdot Q)-(p \cdot q)(P \cdot q)\right](; 3.11) \\
\mathbf{I}^{\prime}\left(\mathcal{Q}_{1}^{2} \mathcal{Q}_{2}^{2}\right) & =-\frac{1}{2} \operatorname{Tr}\left(\mathbb{T}_{1} \mathbb{T}_{2}\right)=(p \cdot P)(q \cdot Q)-(p \cdot Q)(q \cdot P) ; \\
\mathbf{I}^{\prime \prime}\left(\mathcal{Q}_{1}^{2} \mathcal{Q}_{2}^{2}\right) & =-\frac{1}{2} \operatorname{Tr}\left(\mathbb{T}_{12}^{2}\right)=\frac{1}{4}\left[\begin{array}{l}
p^{2} Q^{2}+q^{2} P^{2}+2(p \cdot P)(q \cdot Q) \\
-(p \cdot Q)^{2}-(q \cdot P)^{2}-2(p \cdot q)(P \cdot Q)
\end{array}\right] ; \\
\mathbf{I}_{-1}\left(\mathcal{Q}_{1} \mathcal{Q}_{2}^{3}\right) & \equiv-\frac{1}{2} \operatorname{Tr}\left(\mathbb{T}_{2} \mathbb{T}_{12}\right)=\frac{1}{2}\left[P^{2}(q \cdot Q)+Q^{2}(p \cdot P)-(P \cdot Q)(P \cdot q)-(P \cdot Q)(p \cdot Q)\right] ; \\
\mathbf{I}_{-2}\left(\mathcal{Q}_{2}^{4}\right) & \equiv \mathcal{I}_{4}\left(\mathcal{Q}_{2}\right)=-\frac{1}{2} \operatorname{Tr}\left(\mathbb{T}_{2}^{2}\right)=P^{2} Q^{2}-(P \cdot Q)^{2} .
\end{aligned}
$$

- three $\mathbb{T}$ 's. For $p=2$ centers, there is only one possible non-vanishing invariant polynomial constructed with all possible contractions of three $\mathbb{T}$-tensors out of the ones defined by (3.3)(3.6), namely:

$$
\mathbf{I}_{6}\left(\mathcal{Q}_{1}^{3} \mathcal{Q}_{2}^{3}\right) \equiv-\operatorname{Tr}\left(\mathbb{T}_{1} \mathbb{T}_{2} \mathbb{T}_{12}\right)=-\frac{1}{2}\left[\begin{array}{l}
(q \cdot P)(p \cdot Q)^{2}-(p \cdot Q)(P \cdot q)^{2} \\
+(q \cdot P)(q \cdot Q)(p \cdot P)-(Q \cdot p)(q \cdot Q)(p \cdot P) \\
+(q \cdot P)(p \cdot q)(P \cdot Q)-(Q \cdot p)(p \cdot q)(P \cdot Q) \\
-(q \cdot P) p^{2} Q^{2}+(Q \cdot p) P^{2} q^{2} \\
-(q \cdot Q) P^{2}(p \cdot q)+(q \cdot Q) p^{2}(P \cdot Q) \\
-(p \cdot P) q^{2}(P \cdot Q)+(p \cdot P) Q^{2}(p \cdot q),
\end{array}\right] .
$$

This $G_{4}$-invariant polynomial will turn out to be dependent on the lower-degrees $G_{4}$-invariant polynomials in all $\mathcal{N}=2, d=4$ models $\left(s t u, s t^{2}\right.$ and $\left.t^{3}\right)$ which we consider in the present investigation.

\section{The Role of the Horizontal Symmetry $S L_{h}(2, \mathbb{R})$}

The rank-2 antisymmetric $\mathbb{T}$-tensors (3.3) -(3.6) fit into an irrepr. 3 (spin $s=1$ ) of a further "horizontal" symmetry $S L_{h}(2, \mathbb{R})$, which takes into account the combinatorics under the exchange of the centers $1 \leftrightarrow 2$ (here the subscript " $h$ " stands for "horizontal"). Such a 3 irrepr. is the symmetric part of the tensor product of two fundamental irrepr. 2 ( $\operatorname{spin} s=1 / 2)$ of $S L_{h}(2, \mathbb{R})$, in which $\mathcal{Q}_{1}$ and $\mathcal{Q}_{2}$ sit, with helicity $+1 / 2$ and $-1 / 2$, respectively:

$$
S L_{h}(2, \mathbb{R}): \mathbf{2} \times \mathbf{2}=\stackrel{\left(\mathbb{T}_{12}, \mathbb{T}_{1}, \mathbb{T}_{2}\right)}{\mathbf{3}_{s}}+\mathbb{1}_{a}
$$

where

$$
\mathbb{T}_{a, \Lambda \Sigma}\left(\mathcal{Q}_{1} \mathcal{Q}_{2}\right) \equiv \frac{1}{2}\left(q_{\Lambda} P_{\Sigma}+q_{\Sigma} P_{\Lambda}-p_{\Lambda} Q_{\Sigma}-p_{\Sigma} Q_{\Lambda}\right)
$$

is a rank- 2 symmetric tensor, which is antisymmetric under $1 \leftrightarrow 2$, and thus it vanishes for $1 \equiv 2$. Note that under $1 \leftrightarrow 2 \mathbb{T}_{12}$ is invariant, whereas $\mathbb{T}_{1} \leftrightarrow \mathbb{T}_{2}$.

From the definitions (3.12) and (3.13), the squared norm of the 3 -vector $\mathbb{T} \equiv\left(\mathbb{T}_{1}, \mathbb{T}_{12}, \mathbb{T}_{2}\right)$ reads

$$
\|\mathbb{T}\|^{2} \equiv-\frac{1}{2} \operatorname{Tr}\left(\mathbb{T}_{1} \mathbb{T}_{2}\right)+\frac{1}{2} \operatorname{Tr}\left(\mathbb{T}_{12}^{2}\right)=\mathbf{I}^{\prime}-\mathbf{I}^{\prime \prime}
$$

This is a singlet of $S L_{h}(2, \mathbb{R})$, symmetric under the center exchange $1 \leftrightarrow 2$. 
Also the subscripts of the the four $G_{4}$-invariants $\mathbf{I}_{+2}, \mathbf{I}_{+1}, \mathbf{I}_{-1}$ and $\mathbf{I}_{-2}$, defined by (3.10), (3.11), (3.14) and (3.15), denote their helicity with respect to the relevant irrepr. of the horizontal symmetry $S L_{h}(2, \mathbb{R})$. Indeed, by further defining

$$
\mathbf{I}_{0} \equiv \frac{1}{3}\left(\mathbf{I}^{\prime}+2 \mathbf{I}^{\prime \prime}\right)
$$

the five $G_{4}$-invariants $\mathbf{I}_{+2}, \mathbf{I}_{+1}, \mathbf{I}_{0}, \mathbf{I}_{-1}$ and $\mathbf{I}_{-2}$ sit in the $\mathbf{5}$ (spin $s=2$ ) irrepr. of $S L_{h}(2, \mathbb{R})$ itself:

$$
\mathfrak{I} \equiv \stackrel{\operatorname{spin}}{\mathbf{5}} \boldsymbol{5}=2 \equiv\left(\begin{array}{lllll}
\mathbf{I}_{+2}, & \mathbf{I}_{+1}, & \mathbf{I}_{0}, & \mathbf{I}_{-1}, & \mathbf{I}_{-2}
\end{array}\right) ; \operatorname{Tr} \mathfrak{I}=0 .
$$

The very definitions (3.10)-(3.15) and (4.4) characterize the 5 given in (4.5) as a part symmetric tensor product of two irreprs. 3 of $S L_{h}(2, \mathbb{R})$ itself (in which the $\mathbb{T}$-tensors (3.3)-(3.6) sit):

$$
S L_{h}(2, \mathbb{R}): \mathbf{3} \times \mathbf{3}=\stackrel{\left(\mathbf{I}_{+2}, \mathbf{I}_{+1}, \mathbf{I}_{0}, \mathbf{I}_{-1}, \mathbf{I}_{-2}\right)}{\mathbf{5}_{s}}+\underset{\mathbf{1}}{\mathbf{T}}+\mathbf{3}_{a} .
$$

Note that the $S L_{h}(2, \mathbb{R})$-singlet $\mathbf{T}$ defined in (4.3) sits in the $\mathbf{1}_{s}$ in the right-hand side of decomposition (4.6).

Notice that all the $G_{4}$-quartic invariants $\mathbf{I}_{+2}, \mathbf{I}_{+1}, \mathbf{I}_{0}, \mathbf{I}^{\prime}, \mathbf{I}^{\prime \prime}, \mathbf{I}_{-1}$ and $\mathbf{I}_{-2}$ consistently reduce to $\mathcal{I}_{4}(\mathcal{Q})$ defined in $(3.9)$ in the 1-center limit $1 \equiv 2$. Furthermore, they satisfy the following sum rule:

$$
\mathcal{I}_{4}\left(\mathcal{Q}_{1}+\mathcal{Q}_{2}\right)=\mathbf{I}_{+2}+4 \mathbf{I}_{+1}+6 \mathbf{I}_{0}+4 \mathbf{I}_{-1}+\mathbf{I}_{-2}
$$

Moreover, under the center exchange $1 \leftrightarrow 2$, the polynomial $\mathbf{I}_{0}$ gets unchanged, whereas

$$
\mathbf{I}_{+2} \leftrightarrow \mathbf{I}_{-2}, \mathbf{I}_{+1} \leftrightarrow \mathbf{I}_{-1} .
$$

One can compute also the following $S L_{h}(2, \mathbb{R})$-singlets:

$$
\begin{aligned}
& \operatorname{Tr}\left(\mathfrak{I}^{2}\right)=\mathbf{I}_{+2} \mathbf{I}_{-2}+3 \mathbf{I}_{0}^{2}-4 \mathbf{I}_{+1} \mathbf{I}_{-1} ; \\
& \operatorname{Tr}\left(\mathfrak{I}^{3}\right)=\mathbf{I}_{0}^{3}+\mathbf{I}_{+2} \mathbf{I}_{-1}^{2}+\mathbf{I}_{-2} \mathbf{I}_{+1}^{2}-\mathbf{I}_{+2} \mathbf{I}_{-2} \mathbf{I}_{0}-2 \mathbf{I}_{+1} \mathbf{I}_{0} \mathbf{I}_{-1} .
\end{aligned}
$$

(4.9) and (4.10) are the only independent $S L_{h}(2, \mathbb{R})$-singlets which can be built out of the $3 \times 3$ symmetric matrix $\mathfrak{I}$ defined in (4.5), due to its very tracelessness. Furthermore, they both vanish in the 1 -center limit $1 \equiv 2$.

Also the polynomial $\mathbf{I}_{6}$ defined by (3.16) is a singlet of the horizontal symmetry $S L_{h}(2, \mathbb{R})$; it is antisymmetric under $1 \leftrightarrow 2$, and it vanishes when $1 \equiv 2$.

The very same properties are shared by the quadratic invariant given by the symplectic product $(\Omega$ denoting here the $S p(8, \mathbb{R})$ metric)

$$
\begin{aligned}
\mathcal{W} & \equiv\left\langle\mathcal{Q}_{1}, \mathcal{Q}_{2}\right\rangle \equiv \mathcal{Q}_{1}^{T} \Omega \mathcal{Q}_{2} \\
& =-p^{0} Q_{0}-p^{1} Q_{1}-p^{2} Q_{2}-p^{3} Q_{3}+q_{0} P^{0}+q_{1} P^{1}+q_{2} P^{2}+q_{3} P^{3}
\end{aligned}
$$

which is nothing but the $\eta$-trace of the antisymmetric $\mathbb{T}$-tensor defined by (4.2):

$$
\mathcal{W}=\eta^{\Lambda \Sigma} \mathbb{T}_{a, \Lambda \Sigma}\left(\mathcal{Q}_{1} \mathcal{Q}_{2}\right) \equiv \operatorname{Tr}\left(\mathbb{T}_{a}\right) .
$$

Thus, $\mathcal{W}$ is an $S L_{h}(2, \mathbb{R})$-singlet, antisymmetric under $1 \leftrightarrow 2$.

By recalling (4.3) and (4.12), a particular combination of $S L_{h}(2, \mathbb{R}$ )-singlets (symmetric under $1 \leftrightarrow 2$ ) which will be relevant in the subsequent treatment can be defined as follows:

$$
\mathcal{X} \equiv 2\|\mathbb{T}\|^{2}-\frac{1}{2} \operatorname{Tr}^{2}\left(\mathbb{T}_{a}\right)=2\left(\mathbf{I}^{\prime}-\mathbf{I}^{\prime \prime}\right)-\frac{1}{2} \mathcal{W}^{2} .
$$


Note that both (4.11) and (4.13) vanish when $1 \equiv 2$.

An equivalent group theoretical characterization of the quartic invariants $\mathbf{I}_{+2}, \mathbf{I}_{+1}, \mathbf{I}_{0}, \mathbf{I}_{-1}$ and $\mathbf{I}_{-2}$ fit them into a rank-4 completely symmetric tensor of the fundamental irrepr. 2 of $S L_{h}(2, \mathbb{R})$ itself.

This interpretation enjoys an immediate generalisation to the case of $p$ centers. Indeed, as mentioned in Sec. 4, in this case the "horizontal" combinatorics symmetry group is $S L_{h}(p, \mathbb{R})$.

As a consequence, the quartic polynomial $G_{4}$-invariants which can be obtained by computing $\mathcal{I}_{4}\left(\sum_{a=1}^{p} \mathcal{Q}_{a}\right)$ sit in the rank-4 completely symmetric tensor product of the fundamental irrepr. $\mathbf{p}$ of $S L_{h}(p, \mathbb{R})$, and their number is thus given by $\left(\begin{array}{c}p+3 \\ 4\end{array}\right)$, which yields 1 for $p=1$ (namely, $\mathcal{I}_{4}(\mathcal{Q})$ ), 5 for $p=2,15$ for $p=3$, etc.

Furthermore, the quadratic polynomial $G_{4}$-invariants (antisymmetric under $1 \leftrightarrow 2$ ) sit in the rank-2 antisymmetric tensor product of the fundamental irrepr. 2 of $S L_{h}(p, \mathbb{R})$, and their number is thus given by $\frac{p(p-1)}{2}$, which yields 0 for $p=1,1$ for $p=2$ (namely, the symplectic product $\mathcal{W} \equiv\left\langle\mathcal{Q}_{1}, \mathcal{Q}_{2}\right\rangle$ ), 3 for $p=3$ (namely, the three symplectic products $\mathcal{W}_{1} \equiv\left\langle\mathcal{Q}_{1}, \mathcal{Q}_{2}\right\rangle, \mathcal{W}_{2} \equiv\left\langle\mathcal{Q}_{1}, \mathcal{Q}_{3}\right\rangle$ and $\mathcal{W}_{3} \equiv\left\langle\mathcal{Q}_{2}, \mathcal{Q}_{3}\right\rangle$ ), et cetera. The very same holds for the sextic $G_{4}$-polynomial invariant $\mathbf{I}_{6}$ defined by (3.16).

\section{Independent Invariants, Constraints and their $s t^{2}$ and $t^{3}$ Descendants}

We now face the issue of the independence of the various $G_{4}$-invariants introduced so far, namely $\mathbf{I}_{+2}, \mathbf{I}_{+1}, \mathbf{I}^{\prime}, \mathbf{I}^{\prime \prime}, \mathbf{I}_{-1}, \mathbf{I}_{-2}, \mathcal{W}$ and $\mathbf{I}_{6}$, which is directly related to the explicit derivation of the various constraints among them.

Generally, an effective method to check the functional relations (if any) holding within a given set of $G_{4}$-invariants is the one based on the analysis of the Jacobian matrix. In the case under consideration, one defines the rectangular $8 \times 16$ Jacobian matrix $\mathbf{J}$

$$
\mathbf{J} \equiv \frac{\partial \mathbf{I}}{\partial \mathbf{Q}^{\alpha}}
$$

where

$$
\mathbf{I} \equiv\left(\mathbf{I}_{+2}, \mathbf{I}_{+1}, \mathbf{I}^{\prime}, \mathbf{I}^{\prime \prime}, \mathbf{I}_{-1}, \mathbf{I}_{-2}, \mathcal{W}, \mathbf{I}_{6}\right)
$$

and $(\alpha=1, \ldots, 16)$

$$
\mathbf{Q}^{\alpha} \equiv\left(\mathcal{Q}_{1}^{T}, \mathcal{Q}_{2}^{T}\right)^{T}=\left(p^{0}, p^{1}, p^{2}, p^{3}, q_{0}, q_{1}, q_{2}, q_{3}, P^{0}, P^{1}, P^{2}, P^{3}, Q_{0}, Q_{1}, Q_{2}, Q_{3}\right)^{T}
$$

is the charge vector spanning the 16-dimensional real vector space

$$
V \equiv V_{1} \oplus V_{2}
$$

where $V_{i}$ is the 8-dimensional irrepr. space of the $(\mathbf{2}, \mathbf{2}, \mathbf{2})$ ( $\left.\operatorname{spin} s=\left(\frac{1}{2}, \frac{1}{2}, \frac{1}{2}\right)\right)$ of the $U$-duality group $[S L(2, \mathbb{R})]^{3}$, in which the magnetic and electric charges of the $\mathrm{BH}$ at center $i=1,2$ sit.

By direct computation, one can check that the rank of the matrix $\mathbf{J}$ is seven; in other words, all minors of rank eight of $\mathbf{J}$ do vanish, whereas all minors of order seven are non-zero.

A first way to explain the rank seven of $\mathbf{J}$ is as follows.

The whole vector space spanned by the charge vector $\mathbf{Q}^{\alpha}(\underline{5.3})$ of the two BH centers in the stu model is given by the 16-dimensional space $V$ defined in (5.4). On the other hand, the generic (BPS) orbit of $\mathbf{Q}^{\alpha}$ is given by $\mathcal{O}=S L(2, \mathbb{R}) \times S O(2,2)$ itself, and thus it is 9-dimensional. Thus, the general formulæ (1.2)-(1.3) yield that

$$
\operatorname{dim}_{\mathbb{R}} V=\operatorname{dim}_{\mathbb{R}} \mathcal{O}+I_{p=2}
$$

where $V$ is spanned by the multi-center charge vector $\mathbf{Q}$ belonging to the multi-center orbit $\mathcal{O}$. Thus, in the $s t u$ model the number of polynomial invariants is $I_{p=2}=16-9=7$, in agreement with the 
computations reported above. One can also check that (1.2) applied to the 1-center case of stu model trivially yields the correct result, namely $I_{p=1}=8-7=1$ (i.e., the quartic invariant (2.8) - in "special coordinates" basis or, equivalently (3.9) - in Calabi-Vesentini basis).

As we will prove in App. A, a polynomial constraint of order 12 relates the eight $[S L(2, \mathbb{R})]^{3}$ invariant polynomials introduced so far, namely:

$$
\mathcal{P}_{12, s t u} \equiv \mathbf{I}_{6}^{2}+\mathcal{W} \mathcal{X} \mathbf{I}_{6}+\operatorname{Tr}\left(\mathfrak{I}^{3}\right)+\frac{\operatorname{Tr}\left(\mathfrak{I}^{2}\right) \mathcal{W}^{2}}{12}-\frac{\operatorname{Tr}\left(\mathfrak{I}^{2}\right) \mathcal{X}}{3}-\frac{\mathcal{W}^{6}}{432}+\frac{\mathcal{W}^{4} \mathcal{X}}{36}+\frac{5 \mathcal{W}^{2} \mathcal{X}^{2}}{36}+\frac{4 \mathcal{X}^{3}}{27}=0
$$

This manifestly $S L_{h}(2, \mathbb{R})$-invariant polynomial constraint makes the counting of independent $G_{4^{-}}$ invariant polynomials perfectly consistent with the result and analysis presented above. Namely, in the $s t u$ model, the eight $[S L(2, \mathbb{R})]^{3}$-invariant polynomials $\mathbf{I}_{+2}, \mathbf{I}_{+1}, \mathbf{I}^{\prime}, \mathbf{I}^{\prime \prime}, \mathbf{I}_{-1}, \mathbf{I}_{-2}, \mathcal{W}$ and $\mathbf{I}_{6}$ are constrained by the 12-degree relation (5.6). Thus, the number of 2 -center independent $[S L(2, \mathbb{R})]^{3}$ invariant polynomials in the $s t u$ model is $I_{p=2}=8-1=7$, in agreement with the result (both from Jacobian analysis and general counting) discussed above.

As discussed in Secs. 6] and 7 (as well as in App. A), the further reduction of the constraint (5.6) to the $s t^{2}$ and $t^{3}$ models give rise to an hierarchy of manifestly $S L_{h}(2, \mathbb{R})$-invariant polynomial relations among the various $G_{4}$-invariants.

\section{The $s t^{2}$ Model}

Through a suitable reduction procedure (see App. A, as well as Sec. 5 of [42]), the stu model gives rise to the so-called $\mathcal{N}=2, d=4 s t^{2}$ model. In the "special coordinates" symplectic frame (see e.g. [45] and Refs. therein), this model is defined by the prepotential

$$
\begin{aligned}
F(X) & \equiv \frac{1}{3 !} d_{i j k} \frac{X^{i} X^{j} X^{k}}{X^{0}}=\frac{X^{1}\left(X^{2}\right)^{2}}{X^{0}}=\left(X^{0}\right)^{2} s t^{2} \Leftrightarrow d_{122}=2 \\
s & \equiv \frac{X^{1}}{X^{0}}, t \equiv \frac{X^{2}}{X^{0}} .
\end{aligned}
$$

The $S p(6, \mathbb{R})$-vector of holomorphic symplectic sections can thus be written as follows:

$$
\mathbf{V} \equiv\left(\begin{array}{c}
X^{0} \\
X^{1} \\
X^{2} \\
F_{0} \\
F_{1} \\
F_{2}
\end{array}\right)=\left(\begin{array}{c}
1 \\
s \\
t \\
-s t^{2} \\
t^{2} \\
2 s t
\end{array}\right) X^{0}=\left(\begin{array}{c}
1 \\
s \\
t \\
-\mathcal{F} \\
\mathcal{F}_{1} \\
\mathcal{F}_{2}
\end{array}\right) X^{0}
$$

Here we will not report a detailed treatment of the $s t^{2}$ model (we address the reader $e . g$. to [42, 46, 47]), we will just confine ourselves to some basics, useful for the developments given below.

The $s t^{2}$ model is the unique example of $d$-SK geometry with $\operatorname{dim}_{\mathbb{C}}=2$ (corresponding to $n_{V}=2$ vector multiplets). It is based on the rank-2 factorised symmetric coset

$$
\frac{G_{4}}{H_{4}}=\frac{S L(2, \mathbb{R})}{U(1)} \times \frac{S O(2,1)}{S O(2)} \sim\left[\frac{S L(2, \mathbb{R})}{U(1)}\right]^{2},
$$

where $G_{4}=[S L(2, \mathbb{R})]^{2}$ is the $d=4 U$-duality group, and $H_{4}=[U(1)]^{2}$ its mcs. This coset, with constant curvature -3 [52], is the first element $(n=1)$ of the infinite sequence of reducible SK symmetric cosets $\frac{S L(2, \mathbb{R})}{U(1)} \times \frac{S O(2, n)}{S O(2) \times S O(n)}$ (the so-called Jordan symmetric sequence; see e.g. [48, 34, and Refs. therein). 
As the $s t u$ model, the $s t^{2}$ model admits all classes of extremal BH attractors [1] (for a general analysis and the treatment of attractor-supporting charge orbits, see e.g. [23]). The BPS solutions were known after [41, 49, whereas the explicit expression of the non-BPS $Z_{H}=0$ attractors have been obtained in [42]. The non-BPS $Z_{H} \neq 0$ attractor solutions can also be obtained, through a "stu $\rightarrow s t^{2}$ reduction" procedure (see e.g. Sect. 5 of [42]), by performing the (near-)horizon limit $(\tau \rightarrow-\infty)$ of the general expressions of the $\frac{1}{2}$-BPS and non-BPS $Z_{H} \neq 0$ attractor flows of the stu model, obtained in full generality in [46] (see also Refs. therein, as well as [47]).

By introducing the $S p(6, \mathbb{R})$-vector of charges

$$
\mathcal{Q} \equiv\left(p^{0}, p^{1}, p^{2}, q_{0}, q_{1}, q_{2}\right)^{T},
$$

in the "special coordinate basis" the unique polynomial invariant (homogeneous and quartic in the charges) of the $(\mathbf{2}, \mathbf{3})$ (namely spin $\left.s=\left(\frac{1}{2}, 1\right)\right)$ of the $U$-duality group $[S L(2, \mathbb{R})]^{2}$ reads

$$
\mathcal{I}_{4}(\mathcal{Q})=-\left(p^{0}\right)^{2} q_{0}^{2}-\left(p^{1}\right)^{2} q_{1}^{2}-2 p^{0} q_{0} p^{1} q_{1}-2 p^{0} q_{0} p^{2} q_{2}+2 p^{1} q_{1} p^{2} q_{2}+4 q_{0} p^{1}\left(p^{2}\right)^{2}-p^{0} q_{1} q_{2}^{2},
$$

which can be obtained from (2.9) by specifying $d_{122}=2$ and $d^{122}=1 / 2$, consistently with the nonlinear relation (2.10).

By considering 2-center extremal BHs in the $s t^{2}$ model, with the charge vectors associated to the two centers respectively reading

$$
\begin{aligned}
& \mathcal{Q}_{1} \equiv\left(p^{0}, p^{1}, p^{2}, q_{0}, q_{1}, q_{2}\right)^{T} ; \\
& \mathcal{Q}_{2} \equiv\left(P^{0}, P^{1}, P^{2}, Q_{0}, Q_{1}, Q_{2}\right)^{T},
\end{aligned}
$$

the eight $G_{4}$-invariant polynomials $\mathbf{I}_{+2}, \mathbf{I}_{+1}, \mathbf{I}^{\prime}, \mathbf{I}^{\prime \prime}, \mathbf{I}_{-1}, \mathbf{I}_{-2}, \mathcal{W}$ and $\mathbf{I}_{6}$ can be obtained from their very definitions (3.10)-(3.15), (4.11) and (3.16) by simply specifying $\Lambda=0,1,2$ (and thus using the metric $\eta_{\Lambda \Sigma}=\eta^{\Lambda \Sigma}$ of $S O(2,1)$ to raise and lower the indices).

In order to establish the independence of such 2-center $[S L(2, \mathbb{R})]^{2}$-invariants introduced above, we will exploit the Jacobian method used above for the stu model, adapted to the model under consideration. To this end, one defines the rectangular $6 \times 12$ Jacobian matrix $\mathbf{J}$ (5.1), where I is defined in (5.2), with $(\alpha=1, \ldots, 12)$

$$
\mathbf{Q}^{\alpha} \equiv\left(\mathcal{Q}_{1}^{T}, \mathcal{Q}_{2}^{T}\right)^{T}=\left(p^{0}, p^{1}, p^{2}, q_{0}, q_{1}, q_{2}, P^{0}, P^{1}, P^{2}, Q_{0}, Q_{1}, Q_{2}\right)^{T}
$$

is the charge vector spanning the 12-dimensional vector space $V$ given by (5.4), where now $V_{i}$ is the 6 -dimensional irrepr. space of the $(\mathbf{2}, \mathbf{3})$ ( $\left.\operatorname{spin} s=\left(\frac{1}{2}, 1\right)\right)$ of the $U$-duality group $[S L(2, \mathbb{R})]^{2}$, in which the magnetic and electric charges of the $\mathrm{BH}$ at center $i=1,2$ sit.

By direct computation, one can check that the rank of the matrix $\mathbf{J}$ for the $s t^{2}$ model is six; in other words, all minors of rank six of $\mathbf{J}$ are non-zero, whereas all minors of rank seven and eight do vanish.

Similarly to the discussion done for the stu model, a simple venue for the explanation for the rank six of $\mathbf{J}$ in the $s t^{2}$ model is as follows.

The whole vector space spanned by the charge vector $\mathbf{Q}^{\alpha}(\underline{6.9})$ of the two $\mathrm{BH}$ centers in the $s t^{2}$ model is given by the 12-dimensional space $V$ defined in (5.4). On the other hand, the generic (BPS) orbit of $\mathbf{Q}^{\alpha}$ is given by $\mathcal{O}=S L(2, \mathbb{R}) \times S O(2,1)$ itself, and thus it is 6-dimensional. Thus, by applying the relation (1.2) (holding in the theory of polynomial invariants of Lie groups) to the $s t^{2}$ model, the final result on the number of polynomial invariants is $I_{p=2}=12-6=6$, in agreement with the computations reported above. One can also check that (1.2) applied to the 1-center case of $s t^{2}$ model trivially yields the correct result, namely $I_{p=1}=6-5=1$ (i.e., the quartic invariant (6.6) - in "special coordinates" basis or, equivalently (3.9) - in Calabi-Vesentini basis). 
The above counting of independent 2-center polynomial invariants of the $U$-duality group $[S L(2, \mathbb{R})]^{2}$ of the $s t^{2}$ model is consistent with the number of independent, manifestly $S L_{h}(2, \mathbb{R})$-invariant polynomial relations holding for the $s t^{2}$ model itself.

Indeed, as we will detail in App. A. starting from the stu model and its constraint (5.6), a suitable reduction to $s t^{2}$ model determines the following two manifestly $S L_{h}(2, \mathbb{R})$-invariant constraints:

$$
\begin{aligned}
\mathcal{P}_{16, s t^{2}} & \equiv \mathcal{P}_{16, t^{3}}-\frac{32}{3} \operatorname{Tr}\left(\mathfrak{I}^{2}\right) \mathcal{X}^{2}+\frac{8}{9} \mathcal{W}^{4} \mathcal{X}^{2}+\frac{64}{27} \mathcal{W}^{2} \mathcal{X}^{3}+\frac{16}{9} \mathcal{X}^{4}=0 \\
\mathcal{P}_{8, s t^{2}} & \equiv-12 \operatorname{Tr}\left(\mathfrak{I}^{2}\right)+24 \mathbf{I}_{6} \mathcal{W}+\left(\mathcal{W}^{2}+2 \mathcal{X}\right)^{2}=0
\end{aligned}
$$

where

$$
\mathcal{P}_{16, t^{3}} \equiv 16 \operatorname{Tr}^{2}\left(\mathfrak{I}^{2}\right)+64 \operatorname{Tr}\left(\mathfrak{I}^{3}\right) \mathcal{W}^{2}+\frac{8}{3} \operatorname{Tr}\left(\mathfrak{I}^{2}\right) \mathcal{W}^{4}-\frac{1}{27} \mathcal{W}^{8} .
$$

Note that (6.11) expresses $\mathbf{I}_{6}$ in terms of the other invariants, whereas (6.10) is the constraint which decreases the number of independent polynomial invariants from seven to six.

It is also worth pointing out that the very structure of constraints (5.6) and (6.12) is determined by the underlying $S L_{h}(2, \mathbb{R})$-invariance; for instance, this latter constrains the inhomogeneous term of (6.12) to be the square of the coefficient of $\mathcal{W}^{4}$ in the same equation. Also, the fact that a term proportional to $\mathcal{W}^{6}$ is missing in Eq. (6.12) is due to the tracelessness of $\mathfrak{I}$ itself (recall (4.5)): $\operatorname{Tr} \mathfrak{I}=0$.

The manifestly $S L_{h}(2, \mathbb{R})$-invariant polynomial constraints (6.11)-6.10) make the counting of independent $G_{4}$-invariant polynomials perfectly consistent with the result and analysis presented above. Namely, in the $s t^{2}$ model, the eight $[S L(2, \mathbb{R})]^{2}$-invariant polynomials $\mathbf{I}_{+2}, \mathbf{I}_{+1}, \mathbf{I}^{\prime}, \mathbf{I}^{\prime \prime}, \mathbf{I}_{-1}, \mathbf{I}_{-2}, \mathcal{W}$ and $\mathbf{I}_{6}$ are constrained by the 8-degree and 16-degree relations respectively given by Eqs. (6.11) and (6.10). Thus, the number of 2 -center independent $[S L(2, \mathbb{R})]^{2}$-invariant polynomials in the $s t^{2}$ model is $I_{p=2}=8-2=6$, in agreement with the result (both from Jacobian analysis and general counting) discussed above.

\section{The $t^{3}$ Model}

Through a suitable reduction procedure (see App. A, as well as Sec. 5 of [42]), the stu model gives rise to the so-called $\mathcal{N}=2, d=4 t^{3}$ model. In the "special coordinates" symplectic frame (see e.g. [45] and Refs. therein), this model is defined by the prepotential

$$
\begin{aligned}
F(X) & \equiv \frac{1}{3 !} d_{i j k} \frac{X^{i} X^{j} X^{k}}{X^{0}}=\frac{\left(X^{1}\right)^{3}}{X^{0}}=\left(X^{0}\right)^{2} t^{3} \Leftrightarrow d_{111}=6 \\
t & \equiv \frac{X^{1}}{X^{0}} .
\end{aligned}
$$

It is worth recalling that the $t^{3}$ model is the unique example of $d$-special Kähler (SK) geometry [34] with $\operatorname{dim}_{\mathbb{C}}=1$ (corresponding to $n_{V}=1$ vector multiplet). It is based on the rank- 1 symmetric coset

$$
\frac{G_{4}}{H_{4}}=\frac{S L(2, \mathbb{R})}{U(1)}
$$

where $G_{4}=S L(2, \mathbb{R})$ is the $d=4 U$-duality group, and $H_{4}=U(1)$ its maximal compact subgroup $(m c s)$. This coset, with constant curvature $-\frac{2}{3}$ [52], is an isolated case within the classification of homogeneous symmetric non-compact SK manifolds (see e.g. 48, 34, and Refs. therein).

Through the definition (7.2) of the projective coordinate $t$, the $S p(4, \mathbb{R})$-vector of holomorphic symplectic sections can thus be written as follows:

$$
\mathbf{V} \equiv\left(\begin{array}{c}
X^{0} \\
X^{1} \\
F_{0} \\
F_{1}
\end{array}\right)=\left(\begin{array}{c}
1 \\
t \\
-t^{3} \\
3 t^{2}
\end{array}\right) X^{0}=\left(\begin{array}{c}
1 \\
t \\
-\mathcal{F} \\
\mathcal{F}_{1}
\end{array}\right) X^{0}
$$


By introducing the $S p(4, \mathbb{R})$-vector of magnetic and electric charges

$$
\mathcal{Q} \equiv\left(p^{0}, p, q_{0}, q\right)^{T}
$$

the unique invariant (homogeneous quartic polynomial in the charges) of the $\mathbf{4}$ ( $\operatorname{spin} s=3 / 2$ ) irrepr. of the $U$-duality group $S L(2, \mathbb{R})$ reads (in the "special coordinates" symplectic frame)

$$
\mathcal{I}_{4}(\mathcal{Q}) \equiv-\left(p^{0}\right)^{2} q_{0}^{2}+\frac{1}{3} p^{2} q^{2}-2 p^{0} q_{0} p q+4 q_{0} p^{3}-\frac{4}{27} p^{0} q^{3}
$$

In general, the sign of $\mathcal{I}_{4}$ is related to the supersymmetry properties of the only two classes of extremal $\mathrm{BH}$ attractors [1] exhibited by the $t^{3}$ model: namely, $\mathcal{I}_{4}>0$ and $\mathcal{I}_{4}<0$ for $\frac{1}{2}$-BPS and non-BPS $Z_{H} \neq 0$ attractor solutions, respectively (see e.g. Appendix II of [23]). The $t^{3}$ model has been the first supergravity model whose Attractor Eqs. have been completely solved. The BPS attractor solution were known after [41, 49], and in [57] also the non-BPS $Z_{H} \neq 0$ attractor solutions were completely determined (see also [58]). It is worth here pointing out that these results can also be obtained, through a "stu $\rightarrow s t^{2} \rightarrow t^{3}$ reduction" procedure (see e.g. Sect. 5 of [42]), by performing the (near-)horizon limit $(\tau \rightarrow-\infty)$ of the general expressions of the $\frac{1}{2}$-BPS and non-BPS $Z_{H} \neq 0$ attractor flow 5 of the stu model, obtained in full generality in [46] (see also Refs. therein, as well as [59]).

Note that (7.6) can be obtained from the general formula (2.9) by specifying $d_{111}=6$ and $d^{111}=$ $2 / 9$, consistently with the non-linear relation (2.10).

Let us now consider 2-center extremal BHs in the $t^{3}$ model, with the charge vectors associated to the two centers respectively reading

$$
\begin{aligned}
& \mathcal{Q}_{1} \equiv\left(p^{0}, p, q_{0}, q\right)^{T} ; \\
& \mathcal{Q}_{2} \equiv\left(P^{0}, P, Q_{0}, Q\right)^{T} .
\end{aligned}
$$

By working in the "special coordinates" symplectic frame, one can write down the components of the 5 ( $\operatorname{spin} s=2$ ) irrepr. of the horizontal symmetry $S L_{h}(2, \mathbb{R})$ by recalling Eqs. (4.7) and (7.6). Then, it is immediate to obtain the following expressions:

$$
\begin{aligned}
\mathbf{I}_{+2}\left(\mathcal{Q}_{1}^{4}\right) \equiv & -\left(p^{0}\right)^{2} q_{0}^{2}+\frac{1}{3} p^{2} q^{2}-2 p^{0} q_{0} p q+4 q_{0} p^{3}-\frac{4}{27} p^{0} q^{3} ; \\
\mathbf{I}_{+1}\left(\mathcal{Q}_{1}^{3} \mathcal{Q}_{2}\right) \equiv & -\frac{1}{2}\left[\left(p^{0}\right)^{2} q_{0} Q_{0}+p^{0} q_{0}^{2} P^{0}\right]+\frac{1}{6}\left(p^{2} q Q+p q^{2} P\right)+3 p^{2} q_{0} P+p^{3} Q_{0} \\
& -\frac{1}{9} p^{0} q^{2} Q-\frac{1}{27} q^{3} P^{0}-\frac{1}{2}\left(p^{0} q_{0} p Q+p^{0} q_{0} q P+p^{0} p q Q_{0}+p q_{0} q P^{0}\right) ; \\
\mathbf{I}_{0}\left(\mathcal{Q}_{1}^{2} \mathcal{Q}_{2}^{2}\right) \equiv & -\frac{1}{6}\left[\left(p^{0}\right)^{2} Q_{0}^{2}+q_{0}^{2}\left(P^{0}\right)^{2}\right]-\frac{2}{3} p^{0} q_{0} P^{0} Q_{0}+\frac{1}{18}\left(p^{2} Q^{2}+q^{2} P^{2}\right)+\frac{2}{9} p q P Q \\
& +2\left(p q_{0} P^{2}+p^{2} P Q_{0}\right)-\frac{2}{27}\left(p^{0} q Q^{2}+q^{2} P^{0} Q\right) \\
& -\frac{1}{3}\left(p^{0} q_{0} P Q+p q P^{0} Q_{0}+p^{0} p Q_{0} Q+q_{0} q P^{0} P+p^{0} q P Q_{0}+p q_{0} P^{0} Q\right) ; \\
\mathbf{I}_{-1}\left(\mathcal{Q}_{1} \mathcal{Q}_{2}^{3}\right) \equiv & -\frac{1}{2}\left[q_{0}\left(P^{0}\right)^{2} Q_{0}+p^{0} P^{0} Q_{0}^{2}\right]+\frac{1}{6}\left(q P^{2} Q+p P Q^{2}\right)+3 p P^{2} Q_{0}+q_{0} P^{3} \\
& -\frac{1}{9} q P^{0} Q^{2}-\frac{1}{27} p^{0} Q^{3}-\frac{1}{2}\left(q P^{0} Q_{0} P+p P^{0} Q_{0} Q+q_{0} P^{0} P Q+p^{0} P Q_{0} Q\right) ; \\
\mathbf{I}_{-2}\left(\mathcal{Q}_{2}^{4}\right)= & -\left(P^{0}\right)^{2} Q_{0}^{2}+\frac{1}{3} P^{2} Q^{2}-2 P^{0} Q_{0} P Q+4 Q_{0} P^{3}-\frac{4}{27} P^{0} Q^{3} .
\end{aligned}
$$

\footnotetext{
${ }^{5}$ Through the "stu $\rightarrow s t^{2} \rightarrow t^{3}$ reduction" procedure, the non-BPS $Z_{H}=0$ attractor flow of stu model consistently degenerates into the $\frac{1}{2}$-BPS attractor flow of the $t^{3}$ model.
} 
In order to establish the independence of such 2-center $S L(2, \mathbb{R})$-invariants, we will exploit the Jacobian method used above for the $s t u$ and $s t^{2}$ models. To this end, one defines the rectangular $6 \times 8$ Jacobian matrix $\mathbf{J}$ (5.1), where I is defined by

$$
\mathrm{I} \equiv\left(\mathbf{I}_{+2}, \mathbf{I}_{+1}, \mathbf{I}_{0}, \mathbf{I}_{-1}, \mathbf{I}_{-2}, \mathcal{W}\right)
$$

and $(\alpha=1, \ldots, 8)$

$$
\mathbf{Q}^{\alpha} \equiv\left(\mathcal{Q}_{1}^{T}, \mathcal{Q}_{2}^{T}\right)^{T}=\left(p^{0}, p, q_{0}, q, P^{0}, P, Q_{0}, Q\right)^{T}
$$

is the charge vector spanning the 8-dimensional vector space (5.4), where $V_{i}$ is the 4-dimensional irrepr. space of the 4 ( $\operatorname{spin} s=3 / 2)$ irrepr. of the $U$-duality group $S L(2, \mathbb{R})$, in which the magnetic and electric charges of the $\mathrm{BH}$ at center $i=1,2$ sit.

By direct computation, one can check that the rank of the matrix $\mathbf{J}$ in the $t^{3}$ model is five. Namely, all minors of rank six of $\mathbf{J}$ do vanish, whereas all minors of rank five are non-zero.

Similarly to the discussion done for the $s t u$ and $s t^{2}$ models, a simple venue for the explanation for the rank five of $\mathbf{J}$ in the $t^{3}$ model is as follows.

The whole vector space spanned by the charge vector $\mathbf{Q}^{\alpha}(7.15)$ of the two $\mathrm{BH}$ centers in the $t^{3}$ model is given by the 8-dimensional space $V$ defined in (5.4). On the other hand, the generic (BPS) orbit of $\mathbf{Q}^{\alpha}$ is given by $\mathcal{O}=S L(2, \mathbb{R})$ itself, and thus it is 3-dimensional. Thus, by applying the relation (1.2) to the $s t^{2}$ model, the final result on the number of polynomial invariants is $I_{p=2}=8-3=5$, in agreement with the computations reported above. One can also check that (1.2) applied to the 1-center case of $t^{3}$ model trivially yields the correct result, namely $I_{p=1}=4-3=1$ (i.e., the quartic invariant (7.6) - in "special coordinates" basis or, equivalently (3.9) - in Calabi-Vesentini basis).

The above counting of independent 2-center polynomial invariants of the $U$-duality group $S L(2, \mathbb{R})$ of the $t^{3}$ model is consistent with the number of independent, manifestly $S L_{h}(2, \mathbb{R})$-invariant polynomial relations holding for the $t^{3}$ model itself.

Before proceeding, it should be remarked that the irreducible (rank-1) $t^{3}$ model is sui generis with respect to the reducible stu (rank-3) and $s t^{2}$ (rank-2) models. Indeed, while the Calabi-Vesentini [44] $\mathbb{T}$-tensor formalism introduced above can be applied to both the $s t u$ and $s t^{2}$, it requires dome further modifications in order to be applied to the $t^{3}$ model. This can essentially be traced back to the fact that, while the $s t u$ and $s t^{2}$ models are the first two elements ( $n=2$ and $n=1$, respectively) of the aforementioned $\mathcal{N}=2$ Jordan symmetric sequence, the $t^{3}$ model is an isolated case within the classification of homogeneous symmetric non-compact SK manifolds (see e.g. 48, 34, and Refs. therein). As a consequence, the consistent application of the $\mathbb{T}$-tensor (Calabi-Vesentini) formalism to the $t^{3}$ model requires some ad hoc modifications (leading to a "constrained" Calabi-Vesentini symplectic frame), which are derived and studied in App. B.

As we will detail in App. A, starting from the stu model and its constraint (5.6), a suitable reduction to the $t^{3}$ model determines the following two manifestly $S L_{h}(2, \mathbb{R})$-invariant constraints (recall (4.2), (4.12) and (4.13)):

$$
\begin{aligned}
\mathcal{X} & =0 \Leftrightarrow\|\mathbb{T}\|^{2}=\frac{1}{4} \mathcal{W}^{2}=\frac{1}{4} \operatorname{Tr}^{2}\left(\mathbb{T}_{a}\right) ; \\
\mathcal{P}_{8, t^{3}} & \equiv 24 \mathbf{I}_{6} \mathcal{W}-12 \operatorname{Tr}\left(\mathfrak{I}^{2}\right)+\mathcal{W}^{4}=0,
\end{aligned}
$$

and then (recall (6.12) $)$

$$
\mathcal{P}_{16, t^{3}}=0 .
$$

Note that the constraints (6.10) and (7.18) can be seen as a quartic algebraic equation in $\mathcal{W}^{2}$, and it can be checked that only one real positive out of the four generally complex roots exists in the case, thus uniquely matching the square of the symplectic product of $\mathcal{Q}_{1}$ and $\mathcal{Q}_{2}$.

Since $\mathbf{I}_{+2}, \mathbf{I}_{+1}, \mathbf{I}^{\prime}, \mathbf{I}^{\prime \prime}, \mathbf{I}_{-1}$ and $\mathbf{I}_{-2}$ all reduce to $\mathcal{I}_{4}(\mathcal{Q})$, and $\mathcal{W}$ and $\mathcal{X}$ both vanish in the 1-center limit $1 \equiv 2$, it is immediate to check that all above constraints identically vanish in such a limit. Also 
notice that all above constraints gets greatly simplified when $\mathcal{W}=0$ (namely, for mutually local charge vectors $\mathcal{Q}_{1}$ and $\mathcal{Q}_{2}$ ).

The manifestly $S L_{h}(2, \mathbb{R})$-invariant polynomial constraints (7.16)-(7.18) make the counting of independent $G_{4}$-invariant polynomials perfectly consistent with the result and analysis presented above. Namely, in the $t^{3}$ model, the eight $S L(2, \mathbb{R})$-invariant polynomials $\mathbf{I}_{+2}, \mathbf{I}_{+1}, \mathbf{I}^{\prime}, \mathbf{I}^{\prime \prime}, \mathbf{I}_{-1}, \mathbf{I}_{-2}, \mathcal{W}$ and $\mathbf{I}_{6}$ are constrained by the 4-degree, 8-degree and 16-degree relations respectively given by Eqs. (7.16), (7.17) and (7.18). Thus, the number of 2-center independent $S L(2, \mathbb{R})$-invariant polynomials in the $t^{3}$ model is $I_{p=2}=8-3=5$, in agreement with the result (both from Jacobian analysis and general counting) discussed above.

\section{Extension to $S O_{h}^{v}(2,2)$ Symmetry and the Gramian Matrix}

The treatment given in previous Secs. relies on the fact that an $S L_{h}(2, \mathbb{R})$-covariant basis is given by the quintet $\mathfrak{I}$, and the two singlets $\mathcal{W}$ and $\mathcal{X}$ (respectively defined by (4.5), (4.12) and (4.13)). By using such a basis, the following $\left[S L_{h}(2, \mathbb{R}) \times G_{4}\right]$-invariant set of polynomials can be constructed:

$$
\underset{\operatorname{deg}=2}{\mathcal{W}, \underset{\operatorname{deg}=4}{\mathcal{X}}, \operatorname{Tr}\left(\mathfrak{I}^{2}\right),} \underset{\operatorname{deg}=8}{\operatorname{Tr}\left(\mathfrak{I}^{3}\right),},
$$

where the degree in charges has been indicated.

However, a lower degree $\left[S L_{h}(2, \mathbb{R}) \times G_{4}\right]$-invariant polynomial, namely $\mathbf{I}_{6}$ defined by $(3.16)$, is related to $\operatorname{Tr}\left(\mathfrak{I}^{3}\right)$ through the degree-12 polynomial constraints (5.6). Actually, if in (8.1) $\operatorname{Tr}\left(\mathfrak{I}^{3}\right)$ is replaced by $\mathbf{I}_{6}$, one obtains the following complete set of $\left[S L_{h}(2, \mathbb{R}) \times G_{4}\right]$-invariant, with "minimal" degrees in charges (indicated by subscripts):

$$
\underset{\operatorname{deg}=2}{\mathcal{W}}, \underset{\operatorname{deg}=4}{\mathcal{X}}, \underset{\operatorname{deg}=6}{\mathbf{I}_{6}}, \underset{\operatorname{deg}=8}{\operatorname{Tr}\left(\mathfrak{I}^{2}\right)}
$$

Then, by the theory of invariant polynomials of classical Lie groups, one is guaranteed that any other higher-order invariant is related to the lowest-degree invariants by an algebraic relation.

As given by Eqs. (8.12)-(8.15) below, the set (8.2) is naturally related to the symmetry

$$
S O_{h}^{v}(2,2) \equiv S L_{h}(2, \mathbb{R}) \times S L_{v}(2, \mathbb{R}) \stackrel{\mathbb{C}}{\sim} S O_{h}^{v}(4, \mathbb{C}),
$$

which is the direct product of the "horizontal" group $S L_{h}(2, \mathbb{R})$ introduced in Sec. 4 and of the $S L_{v}(2, \mathbb{R})$ factor (the upperscript " $v$ " stands for "vertical") in the $d=4 U$-duality group $G_{4}$ (characterising the three models $s t u, s t^{2}$ and $t^{3}$ treated above, as well as the whole infinite sequences (1.1) and (9.1); see Sec. 9). The last step in (8.3) denotes the isomorphism with the complex group $S O(4, \mathbb{C})$. In the following treatment, we will work with complex groups, thus $S L(2, \mathbb{R}) \stackrel{\mathbb{C}}{\sim} S L(2, \mathbb{C})$ and $S O(2,2) \stackrel{\mathbb{C}}{\sim} S O(4, \mathbb{C})$ and, where denoted, we will then perform the suitable Wick rotation to get the appropriate real form.

In order to highlight the relation between the set (8.2) and the symmetry group $S O_{h}^{v}(4, \mathbb{C})$ defined in (8.3), it is convenient to introduce the $4 \times 4$ complex symmetric, manifestly $S O_{h}^{v}(4, \mathbb{C})$-covariant so-called Gramian matrix [60]

$$
\mathbf{G} \equiv\left(\begin{array}{cccc}
z_{0}^{2} & z_{0} \cdot z_{1} & z_{0} \cdot z_{2} & z_{0} \cdot z_{3} \\
z_{0} \cdot z_{1} & z_{1}^{2} & z_{1} \cdot z_{2} & z_{1} \cdot z_{3} \\
z_{0} \cdot z_{2} & z_{1} \cdot z_{2} & z_{2}^{2} & z_{2} \cdot z_{3} \\
z_{0} \cdot z_{3} & z_{1} \cdot z_{3} & z_{2} \cdot z_{3} & z_{3}^{2}
\end{array}\right)
$$

where

$$
\left(\begin{array}{c}
z_{0, \Lambda} \\
z_{1, \Lambda} \\
z_{2, \Lambda} \\
z_{3, \Lambda}
\end{array}\right) \equiv \frac{1}{2}\left(\begin{array}{cccc}
1 & 0 & 0 & 1 \\
0 & -i & -i & 0 \\
0 & 1 & -1 & 0 \\
-i & 0 & 0 & +i
\end{array}\right)\left(\begin{array}{c}
p_{\Lambda} \\
q_{\Lambda} \\
P_{\Lambda} \\
Q_{\Lambda}
\end{array}\right)
$$


and the squared norms, scalar products and index raising and lowering are defined through the suitable $S O(2, n)$-metrics $\eta_{\Lambda \Sigma}$ and $\eta^{\Lambda \Sigma}$ (in $\mathcal{N}=4$ theory, $S O(2, n)$ is replaced by $S O(6, n)$; see Eq. (9.1) below).

Then, by denoting the eigenvalues of $\mathbf{G}$ with $\lambda_{i}(i=1, \ldots, 4)$, the characteristic equation of $\mathbf{G}$ reads:

$$
\operatorname{det}(\mathbf{G}-\lambda \mathbb{I})=\prod_{i=1}^{4}\left(\lambda-\lambda_{i}\right)=\lambda^{4}+a \lambda^{3}+b \lambda^{2}+c \lambda+d=0 .
$$

As proved in App. C, the characteristic equation can be used as a generating function for manifestly $S O_{h}^{v}(4, \mathbb{C})$-invariant polynomials. Indeed, by recalling the Newton's identities [61], one can compute that $(a, b, c, d \in \mathbb{R}$; see also [22])

$$
\begin{aligned}
a & \equiv-\operatorname{Tr} \mathbf{G}=-\left(\lambda_{1}+\lambda_{2}+\lambda_{3}+\lambda_{4}\right) \\
b & \equiv \frac{1}{2}\left[(\operatorname{Tr} \mathbf{G})^{2}-\operatorname{Tr}\left(\mathbf{G}^{2}\right)\right]= \\
& =\lambda_{1} \lambda_{2}+\lambda_{1} \lambda_{3}+\lambda_{1} \lambda_{4}+\lambda_{2} \lambda_{3}+\lambda_{2} \lambda_{4}+\lambda_{3} \lambda_{4} ; \\
c & \equiv-\frac{1}{6}\left[(\operatorname{Tr} \mathbf{G})^{3}+2 \operatorname{Tr}\left(\mathbf{G}^{3}\right)-3 \operatorname{Tr}\left(\mathbf{G}^{2}\right) \operatorname{Tr} \mathbf{G}\right]= \\
& =-\left(\lambda_{1} \lambda_{2} \lambda_{3}+\lambda_{1} \lambda_{2} \lambda_{4}+\lambda_{1} \lambda_{3} \lambda_{4}+\lambda_{2} \lambda_{3} \lambda_{4}\right) ; \\
d & \equiv \frac{1}{4}\left[\frac{1}{6}(\operatorname{Tr} \mathbf{G})^{4}+\frac{1}{2}\left[\operatorname{Tr}\left(\mathbf{G}^{2}\right)\right]^{2}+\frac{4}{3} \operatorname{Tr}\left(\mathbf{G}^{3}\right) \operatorname{Tr} \mathbf{G}\right]= \\
& =\operatorname{Tr}\left(\mathbf{G}^{4}\right)-\operatorname{Tr}\left(\mathbf{G}^{2}\right)(\operatorname{Tr} \mathbf{G})^{2}
\end{aligned}
$$

By computing $\operatorname{Tr} \mathbf{G}, \operatorname{Tr}\left(\mathbf{G}^{2}\right), \operatorname{Tr}\left(\mathbf{G}^{3}\right)$ and $\operatorname{Tr}\left(\mathbf{G}^{4}\right)$, and then performing the Wick rotation

$$
\left\{\begin{array}{l}
z_{1, \Lambda} \longrightarrow i z_{1, \Lambda} \\
z_{3, \Lambda} \longrightarrow i z_{3, \Lambda}
\end{array}\right.
$$

in order to switch from $S O_{h}^{v}(4, \mathbb{C})$ back to $S O_{h}^{v}(2,2)$, the following results can be achieved:

$$
\begin{aligned}
\operatorname{Tr} \mathbf{G} & =-\mathcal{W} ; \\
\operatorname{Tr}\left(\mathbf{G}^{2}\right) & =\frac{1}{2}\left(\mathcal{W}^{2}-2 \mathcal{X}\right) ; \\
\operatorname{Tr}\left(\mathbf{G}^{3}\right) & =\frac{1}{4}\left(6 \mathbf{I}_{6}-\mathcal{W}^{3}+6 \mathcal{W} \mathcal{X}\right) ; \\
\operatorname{Tr}\left(\mathbf{G}^{4}\right) & =-\frac{1}{48}\left[12 \operatorname{Tr}\left(\mathfrak{I}^{2}\right)+72 \mathbf{I}_{6} \mathcal{W}-7 \mathcal{W}^{4}+68 \mathcal{W}^{2} \mathcal{X}-28 \mathcal{X}^{2}\right] .
\end{aligned}
$$

Thus, by virtue of Eq. (8.10), it follows that (recall definition (6.11))

$$
\begin{aligned}
\operatorname{det} \mathbf{G} & =\frac{1}{16 \cdot 12}\left[12 \operatorname{Tr}\left(\mathfrak{I}^{2}\right)-24 \mathbf{I}_{6} \mathcal{W}-\left(\mathcal{W}^{2}+2 \mathcal{X}\right)^{2}\right]=-\frac{1}{16 \cdot 12} \mathcal{P}_{8} ; \\
\operatorname{det}(\mathbf{G}-\lambda \mathbb{I}) & =\lambda^{4}+\mathcal{W} \lambda^{3}+\|\mathbb{T}\|^{2} \lambda^{2}-\frac{1}{2} \mathbf{I}_{6} \lambda+\operatorname{det} \mathbf{G}=0 .
\end{aligned}
$$

The relations (8.12)-(8.15) establish the connection between the set (8.2) and the set of manifestly $S O_{h}^{v}(2,2)$-invariant polynomials $\left(\operatorname{Tr} \mathbf{G}, \operatorname{Tr}\left(\mathbf{G}^{2}\right), \operatorname{Tr}\left(\mathbf{G}^{3}\right), \operatorname{Tr}\left(\mathbf{G}^{4}\right)\right)$ in the eigenvalues $\lambda_{i}$ 's (or, equivalently, in the charges $\mathcal{Q}_{1}$ and $\mathcal{Q}_{2}$ ). As proved in App. Cl such four polynomials form a complete basis for the $S O_{h}^{v}(2,2)$-invariant polynomials of the symmetric matrix $\mathbf{G}$. 
1. In the $s t u$ model (and in the $\mathcal{N}=2$ Jordan symmetric sequence for $n \geqslant 3$, as well as in the whole $\mathcal{N}=4$ infinite sequence (9.1) for $n \geqslant 0$ ) there are no relations among the four eigenvalues $\lambda_{i}$ 's (generally, $\mathbf{G}$ has rank 4).

2. In the $s t^{2}$ model, since the charges can be arranged as an $S L_{v}(2, \mathbb{R})$-doublet of $S O(2,1)$-vectors, the $4 \times 4$ matrix $\mathbf{G}$ has non-maximal rank 3, and thus its determinant vanishes. From Eq. (8.10), this yields the following degree- 8 constraint:

$$
\operatorname{det} \mathbf{G} \equiv d=0 \Leftrightarrow \frac{1}{6}(\operatorname{Tr} \mathbf{G})^{4}+\frac{1}{2}\left[\operatorname{Tr}\left(\mathbf{G}^{2}\right)\right]^{2}+\frac{4}{3} \operatorname{Tr}\left(\mathbf{G}^{3}\right) \operatorname{Tr} \mathbf{G}-\operatorname{Tr}\left(\mathbf{G}^{4}\right)-\operatorname{Tr}\left(\mathbf{G}^{2}\right)(\operatorname{Tr} \mathbf{G})^{2}=0,
$$

implying the vanishing of one eigenvalue of $\mathbf{G}$ itself. Eq. (8.18) is an equivalent, manifestly $S O_{h}^{v}(2,2)$-invariant re-writing of the degree-8 polynomial constraint (6.11), which can be used to eliminate e.g. the highest-degree $\left[S L_{h}(2, \mathbb{R}) \times G_{4}\right]$-invariant of the set (8.2), namely the 8degree $\operatorname{Tr}\left(\mathfrak{I}^{2}\right)$, in terms of the other lower-degree invariants, thus reducing (8.2) to the following set of three:

$$
\underset{\operatorname{deg}=2}{\mathcal{W}}, \underset{\operatorname{deg}=4}{\mathcal{X}}, \quad \underset{\operatorname{deg}=6}{\mathbf{I}_{6}}
$$

By using results (8.12)-(8.15) and Eqs. (4.12)-(4.13), the cubic characteristic equation of $\mathbf{G}$ for $s t^{2}$ model can be computed to read

$$
\operatorname{det}(\mathbf{G}-\lambda \mathbb{I})=\prod_{i=1}^{3}\left(\lambda-\lambda_{i}\right)=\lambda^{3}+\mathcal{W} \lambda^{2}+\|\mathbb{T}\|^{2} \lambda-\frac{1}{2} \mathbf{I}_{6}=0 .
$$

3. In the $t^{3}$ model it further holds that (recall Eq. (7.16) )

$$
\mathcal{X}=0 \Leftrightarrow\|\mathbb{T}\|^{2}=\frac{1}{4} \mathcal{W}^{2} .
$$

Consequently, the set (8.19) further reduces down to

$$
\underset{\operatorname{deg}=2}{\mathcal{W}}, \quad \stackrel{\mathbf{I}_{6}}{\operatorname{deg}=6},
$$

and the cubic characteristic equation of $\mathbf{G}$ for $t^{3}$ model can be obtained from (8.20) by implementing the further condition (8.21):

$$
\operatorname{det}(\mathbf{G}-\lambda \mathbb{I})=\prod_{i=1}^{3}\left(\lambda-\lambda_{i}\right)=\lambda\left(\lambda+\frac{1}{2} \mathcal{W}\right)^{2}-\frac{1}{2} \mathbf{I}_{6}=0 .
$$

Notice that in this model the rank of $\mathbf{G}$ is still 3 .

As mentioned above, the constraint relating the $\left[S L_{h}(2, \mathbb{R}) \times G_{4}\right]$-invariants $\operatorname{Tr}\left(\mathfrak{I}^{2}\right)$ and $\operatorname{Tr}\left(\mathfrak{I}^{3}\right)$ to the lower-degree invariant polynomials are given by Eqs. (5.6) and (6.11). Note that, as also discussed in Sec. 7, by eliminating $\mathbf{I}_{6}$ in terms of $\operatorname{Tr}\left(\mathfrak{I}^{3}\right)$ increases the degree in charges of the resulting polynomial constraints, from the degree-12 of (5.6) to the degree-16 of (6.10) and (6.12).

\section{Generalization to $\mathcal{N}=2$ Jordan Symmetric Sequence and $\mathcal{N}=4$ Theory}

Two infinite sequences of $d=4$ supergravity theories exhibit a factorised $U$-duality group and symmetric (vector multiplets') scalar manifold, namely the Jordan symmetric sequence (1.1) and the $\mathcal{N}=4$ (generally matter-coupled [62, 63]) theory $(n \in \mathbb{N} \cup\{0\})$

$$
\mathcal{N}=4: \frac{S L(2, \mathbb{R})}{U(1)} \times \frac{S O(6, n)}{S O(6) \times S O(n)}\left(\mathbb{R} \oplus \boldsymbol{\Gamma}_{5, n-1}\right)
$$


where (1.1) is usually referred to as Jordan symmetric sequence [35, 36], and the round brackets in the right-hand sides denote the corresponding reducible degree-3 Euclidean Jordan algebras [35, 36]. The number $n_{V}$ of matter (vector) multiplets is given by $n+1$ in (1.1) and by $n$ in (9.1).

The $s t u$ and $s t^{2}$ models respectively are the second $(n=2)$ and the first $(n=1)$ elements of the sequence (1.1).

The result $I_{p=2}=7$ obtained for the $s t u$ model in Sec. 2 can be proved to hold for the $\mathcal{N}=2$ Jordan symmetric sequence (1.1) with $n \geqslant 2$, and for the $\mathcal{N}=4$ theory coupled to any number $n \in \mathbb{N}$ of matter (vector) multiplets.

Indeed, the $p=2$-center "large" orbits with $\mathcal{I}_{4}\left(\mathcal{Q}_{1}\right)>0, \mathcal{I}_{4}\left(\mathcal{Q}_{2}\right)>0$ for the sequences (1.1) and (9.1) respectively read:

$$
\begin{aligned}
& \mathbb{R} \oplus \boldsymbol{\Gamma}_{1, n-1}:\left\{\begin{array}{l}
n=0,1,2: S L(2, \mathbb{R}) \times S O(2, n), \text { BPS }(n=0,1,2), \text { non-BPS }(n=1,2) ; \\
n \geqslant 3: S L(2, \mathbb{R}) \times \frac{S O(2, n)}{S O(n-2)}, \text { BPS, non-BPS; } \\
n \geqslant 4: S L(2, \mathbb{R}) \times \frac{S O(2, n)}{S O(1, n-3)}, \text { non-BPS; } \\
n \geqslant 5: S L(2, \mathbb{R}) \times \frac{S O(2, n)}{S O(2, n-4)}, \text { non-BPS; } \\
\mathbb{R} \oplus \boldsymbol{\Gamma}_{5, n-1}:\left\{\begin{array}{l}
n \geqslant 0: S L(2, \mathbb{R}) \times \frac{S O(6, n)}{S O(2, n)}, \frac{1}{4}-\mathrm{BPS} ; \\
n \geqslant 1: S L(2, \mathbb{R}) \times \frac{S O(6, n)}{S O(3, n-1)}, \frac{1}{4}-\mathrm{BPS} ; \\
n \geqslant 3: S L(2, \mathbb{R}) \times \frac{S O(6, n)}{S O(5, n-3)}, \text { non-BPS; }
\end{array}\right. \\
n \geqslant 4: S L(2, \mathbb{R}) \times \frac{S O(6, n)}{S O(6, n-4)}, \text { non-BPS; }
\end{array}\right.
\end{aligned}
$$

By comparing these results with their $p=1$ counterparts [24, 25, 28], one can realize that the 2-center stabilizer is always contained into the 1-center stabiliser with corresponding supersymmetry-preserving properties. Furthermore, the first line of (9.2) summarizes the results of Sects. 2-7] on the stu model $(n=2)$ and its rank-2 and rank-3 descendants, namely the $s t^{2}$ model $(n=1)$ and the $t^{3}$ model $(n=0)$. Since this latter does not belong to the Jordan symmetric sequence, but it is rather an isolated case in the classification of symmetric special Kähler manifolds (see e.g. 34] and Refs. therein), the notation for $n=0$ in the first line of (9.2) is only of formal nature.

The various orbits of (9.2) and (9.3) can be related to the possible choices of signs of the four eigenvalues $\lambda_{1}, \ldots, \lambda_{4}$ of the Gramian matrix $\mathbf{G}$ introduced in Sect. 8 .

Moreover, (9.2) and (9.3) yield that the $\mathcal{N}=2$ BPS orbit with $n=6$ matches the $\mathcal{N}=4$ non-BPS orbit with $n=2$, as well as the $\mathcal{N}=2$ non-BPS orbit with $n=6$ matches the $\mathcal{N}=4 \frac{1}{4}$-BPS orbit with $n=2$. This can be traced back to the fact that the corresponding theories share the very same bosonic sector 64.

Furthermore, it is worth remarking that the $n=0$ case of the $\mathcal{N}=2$ Jordan symmetric sequence (1.1) is nothing but the $\mathcal{N}=2$ axion-dilaton model (truncation of the "pure" $\mathcal{N}=4$ supergravity theory [33]), whose 2-center split flow and marginal stability have been recently studied (in a manifestly $U(1,1)$-covariant symplectic frame) in 37 .

Remarkably, the general formulæ (1.2)-(1.3) yield that the number of independent $G_{4}$-invariant 
polynomials in both reducible sequences (1.1) and (9.1) is $n$-independent and it amounts to $I_{p=2}=7$ :

$$
\begin{aligned}
\mathbb{R} \oplus \boldsymbol{\Gamma}_{1, n-1}(n \geqslant 2) \quad: \quad I_{p=2}=4(2+n)-3-\frac{(2+n)(1+n)}{2}+\frac{(n-2)(n-3)}{2}=7 ; \\
\mathbb{R} \oplus \boldsymbol{\Gamma}_{5, n-1}(n \in \mathbb{N} \cup\{0\}) \quad: \quad I_{p=2}=4(6+n)-3-\frac{(6+n)(5+n)}{2}+\frac{(n+2)(n+1)}{2}=7 .
\end{aligned}
$$

Note that the symmetry (8.3) extends to $S O_{h}^{v}(2,2) \times S O(2, n)$ and $S O_{h}^{v}(2,2) \times S O(6, n)$ for the sequences (1.1) and (9.1), respectively.

In a forthcoming investigation [65, the analysis of 2-center orbits and polynomial $G_{4}$-invariants will be extended to the $d=4$ supergravity theories based on irreducible rank-3 Euclidean Jordan algebras, namely to $\mathcal{N}=2$ "magic" models and to $\mathcal{N}=5,6,8$ supergravities.

\section{Acknowledgments}

We would like to thank Prof. V. S. Varadarajan and Prof. M. Trigiante for enlightening discussions.

E. O. would like to thank CERN Theory Division for hospitality.

The work of S. F. is supported by the ERC Advanced Grant no. 226455, "Supersymmetry, Quantum Gravity and Gauge Fields" (SUPERFIELDS) and in part by DOE Grant DE-FG03-91ER40662.

The work of E. O. and A. Y. is supported by the ERC Advanced Grant no. 226455, "Supersymmetry, Quantum Gravity and Gauge Fields" (SUPERFIELDS).

\section{A Derivation of the Hierarchy of Constraints for $s t u, s t^{2}$ and $t^{3}$ Models}

This Appendix details the derivation of the polynomial constraint (5.6) (or, equivalently, (5.6)) for the stu model. As mentioned above, the further reduction of the constraint (5.6) to the $s t^{2}$ and $t^{3}$ models give rise to an hierarchy of manifestly $S L_{h}(2, \mathbb{R})$-invariant polynomial relations among the various $G_{4}$-invariant polynomials for these models.

We work in the "special coordinates" symplectic frame. In order to highlight the actual dependence of the $G_{4}$-invariant polynomials on the charges $\mathcal{Q}_{1}$ and $\mathcal{Q}_{2}$ themselves, we start and define some charge variables invariant under the $S O(1,1)$ rescaling symmetry of $G_{4}$ [34]. Indeed, by recalling that the components of $\mathcal{Q}_{1}$ and $\mathcal{Q}_{2}$ have the following $S O(1,1)$-weights $(i=1,2,3)$ :

$$
\begin{array}{ccccc}
S O(1,1): & +3 & +1 & -3 & -1 \\
\mathcal{Q}_{1}= & \left(p^{0},\right. & p^{i}, & q_{0}, & \left.q_{i}\right) ; \\
S O(1,1): & +3 & +1 & -3 & -1 \\
\mathcal{Q}_{2}= & \left(P^{0},\right. & P^{i}, & Q_{0}, & \left.Q_{i}\right),
\end{array}
$$

one can define

$$
\begin{aligned}
& \mathbf{x} \equiv q_{1} p^{1}, \mathbf{y} \equiv q_{2} p^{2}, \mathbf{z} \equiv q_{3} p^{3}, \mathbf{r} \equiv q_{0} p^{0} \\
& \mathbf{X} \equiv Q_{1} P^{1}, \mathbf{Y} \equiv Q_{2} P^{2}, \mathbf{Z} \equiv Q_{3} P^{3}, \mathbf{R} \equiv Q_{0} P^{0} \\
& \mathbf{Z}_{1} \equiv Q_{0} P^{1} P^{2} P^{3}, \quad \mathbf{z}_{1} \equiv q_{0} p^{1} p^{2} p^{3} \\
& \mathbf{u} \equiv \frac{q_{0}}{Q_{0}}, \mathbf{u}_{2} \equiv \frac{p^{2}}{P^{2}}, \mathbf{u}_{3} \equiv \frac{p^{3}}{P^{3}}
\end{aligned}
$$

It can be explicitly shown that the eight polynomial $[S L(2, \mathbb{R})]^{3}$-invariants $\mathbf{I}_{+2}, \mathbf{I}_{+1}, \mathbf{I}^{\prime}, \mathbf{I}^{\prime \prime}, \mathbf{I}_{-1}, \mathbf{I}_{-2}$, $I_{6}$ and $\mathcal{W}$ of the stu model can be re-written only in terms of the thirteen $S O(1,1)$-invariant charge variables defined by (A.3). 
As stated at the start of Sec. 5, all minors of order 8 of the relevant Jacobian matrix $\mathbf{J}$ defined by (5.1), (5.2) and (5.3) do vanish. On the other hand, all minors of order 7 are non-zero. This is a compelling evidence that the number of independent $[S L(2, \mathbb{R})]^{3}$-invariant polynomials is seven.

Thus, a (polynomial) relation constraining the aforementioned eight invariants is expected to exist. In order to derive it, it is convenient to work in a particularly simple charge configuration, in which some of the $S O(1,1)$-invariant charge variables (A.3) vanish; namely:

$$
p^{1}=q_{1}=p^{2}=q_{2}=p^{3}=q_{3}=0 \Rightarrow \mathbf{x}=\mathbf{z}=\mathbf{y}=\mathbf{z}_{1}=\mathbf{u}_{2}=\mathbf{u}_{3}=\frac{\mathbf{x} \mathbf{y} \mathbf{z}}{\mathbf{z}_{1}}=\frac{\mathbf{z}_{1}}{\mathbf{u}_{2}}=\frac{\mathbf{z}_{1}}{\mathbf{u}_{3}}=0 .
$$

This implies the extremal $\mathrm{BH}$ at center 1 to be non-BPS $Z_{H} \neq 0$ (i.e. $\mathbf{I}_{+2}<0$ ), thus resulting in

$$
\mathbf{r}=\sqrt{-\mathbf{I}_{+2}}
$$

Clearly, these choices imply some loss in generality, but remarkably, as we will see below, the results achieved within such a configuration actually hold for a completely generic 2-center charge configuration.

By plugging (A.4) and (A.5) into the expressions of $\mathbf{I}_{+2}, \mathbf{I}_{+1}, \mathbf{I}^{\prime}, \mathbf{I}^{\prime \prime}, \mathbf{I}_{-1}, \mathbf{I}_{-2}, I_{6}$ and $\mathcal{W}$ of the stu model, one can solve the resulting algebraic Eqs. for $\mathbf{X}, \mathbf{Y}, \mathbf{u}, \mathbf{R}$ and $\mathbf{Z}_{1}$ as follows:

$$
\begin{aligned}
& \mathbf{u}=\frac{2 \mathbf{I}_{+2}}{2 \mathbf{I}_{+1}+\mathcal{W} \sqrt{-\mathbf{I}_{+2}}}, \quad \mathbf{R}=\frac{4 \mathbf{I}_{+1}^{2}+\mathbf{I}_{+2} \mathcal{W}^{2}}{4\left(-\mathbf{I}_{+2}\right)^{3 / 2}} \\
& \mathbf{X}=-\frac{4 \mathbf{I}_{+1}^{2}+\mathbf{I}_{+2}\left(\mathcal{W}^{2}-4 \mathbf{I}^{\prime}\right)}{4\left(-\mathbf{I}_{+2}\right)^{3 / 2}}, \quad \mathbf{Y}=2 \frac{\mathbf{I}_{+2} \mathbf{I}^{\prime \prime}-\mathbf{I}_{+1}^{2}}{\left(-\mathbf{I}_{+2}\right)^{3 / 2}}-\mathbf{Z}, \\
& \mathbf{Z}_{1}=\frac{1}{8 \mathbf{I}_{+2}^{3}}\left\{8 \mathbf{I}_{+1}^{4}+\mathbf{I}_{+2}^{2}\left[2 \mathbf{I}_{-1} \mathbf{I}_{+1}+\mathcal{W}\left(\mathbf{I}_{6}+\mathbf{I}_{-1} \sqrt{-\mathbf{I}_{+2}}-\mathbf{I}^{\prime \prime} \mathcal{W}\right)\right]\right. \\
& \left.-\mathbf{I}_{+2} \mathbf{I}_{-1}\left[2 \mathbf{I}_{6} \sqrt{-\mathbf{I}_{+2}}+\mathbf{I}^{\prime} \mathcal{W} \sqrt{-\mathbf{I}_{+2}}+\mathbf{I}_{+1}\left(2 \mathbf{I}^{\prime}+4 \mathbf{I}^{\prime \prime}-\mathcal{W}^{2}\right)\right]\right\} .
\end{aligned}
$$

By so doing, one ends up with following equation for the remaining charge variable $\mathbf{Z}$ :

$\mathbf{Z}^{2}=\frac{1}{4\left(\mathbf{I}_{+2}\right)^{3}}\left\{4 \mathbf{I}_{+1}^{4}-\mathbf{I}_{-2} \mathbf{I}_{+2}^{3}-8 \mathbf{I}_{+2} \mathbf{I}^{\prime \prime} \mathbf{I}_{+1}^{2}+\mathbf{I}_{+2}^{2}\left[\left(\mathbf{I}^{\prime}-2 \mathbf{I}^{\prime \prime}\right)^{2}+4 \mathbf{I}_{+1} \mathbf{I}_{-1}+2 \mathbf{I}_{6} \mathcal{W}\right]\right\}+2 \frac{\left(\mathbf{I}_{+2} \mathbf{I}^{\prime \prime}-\mathbf{I}_{+1}^{2}\right) \mathbf{Z}}{\left(-\mathbf{I}_{+2}\right)^{3 / 2}}$,

and self-consistency condition given by Eq. (5.6).

Remarkably, when relaxing the conditions (A.4)-(A.5), one can check by direct calculation that Eq. (5.6) holds for a completely general 2-center charge configuration.

In order to reduce the stu model to the $s t^{2}$ model, the following identifications of charges are to be performed (within the positions (A.4)-(A.5)):

$$
P^{3}=P^{2} \equiv P^{2}, \quad Q_{3}=Q_{2} \equiv \frac{Q_{2}}{2} .
$$

This implies $\mathbf{Y}=\mathbf{Z}$, where (A.6) implies

$$
\mathbf{Y}=\mathbf{Z}=\frac{\mathbf{I}_{+2} \mathbf{I}^{\prime \prime}-\mathbf{I}_{+1}^{2}}{\left(-\mathbf{I}_{+2}\right)^{3 / 2}} .
$$

By inserting (A.10) into (A.8), the result (6.11) is achieved. By plugging this latter back into (5.6), the 16-degree constraint (6.10)-(6.12) is obtained.

On the other hand, the reduction of the $s t u$ model down to the $t^{3}$ model entails the following charge identifications:

$$
P^{3}=P^{2}=P^{1} \equiv P, \quad Q_{3}=Q_{2}=Q_{1} \equiv \frac{Q}{3} .
$$


These latter imply $\mathbf{X}=\mathbf{Y}=\mathbf{Z}$, and Eqs. (A.6)-(A.7) thus yield Eq. (7.16). This latter, inserted into Eqs. (6.11) and (6.10), respectively gives (7.17) and (7.18).

Once again, all above results can be checked to hold in a general 2-charge configuration, and therefore they are completely general.

This analysis relates a "minimal" set of BH charges to the "minimal" number of independent $G_{4}$-invariant polynomials discussed in the present paper (see in particular Sec. 5).

Finally, the action of the $d=4 U$-duality group $G_{4}$ on the charge vectors $\mathcal{Q}_{1}$ and $\mathcal{Q}_{2}$ can be summarised as follows:

1. In the rank-3 stu model $\left(G_{4}=[S L(2, \mathbb{R})]^{3}\right)$ there are 7 independent polynomial $G_{4}$-invariants depending on the $13 S O(1,1)$-invariant combinations. Thus, out of the 16 charges composing the charge vector $\mathbf{Q}^{\alpha}$ defined in (5.3), 6 charges can be set to zero by the action of the nondilatational 6 generators of $G_{4}$, spanning $\left[\frac{S L(2, \mathbb{R})}{S O(1,1)}\right]^{3}$ (namely, one conformal boost and one translational generator for each of the three $\frac{S L(2, \mathbb{R})}{S O(1,1)}$ factors). By recalling the definitions (A.3) of the $S O(1,1)$-invariant charge variables, a representative of a "minimal" charge configuration is e.g. given by

$$
\begin{aligned}
\mathcal{Q}_{1} & =\left(\frac{\mathbf{r}}{\mathbf{u} \mathbf{Z}_{1}} P^{1} P^{2} P^{3}, \frac{\mathbf{z}_{1}}{\mathbf{Z}_{1} \mathbf{u} \mathbf{u}_{2} \mathbf{u}_{3}} P^{1}, \mathbf{u}_{\mathbf{2}} P^{2}, \mathbf{u}_{\mathbf{3}} P^{3}, \frac{\mathbf{u} \mathbf{Z}_{1}}{P^{1} P^{2} P^{3}}, \frac{\mathbf{x} \mathbf{Z}_{1}}{\mathbf{z}_{1}} \frac{\mathbf{u} \mathbf{u}_{2} \mathbf{u}_{3}}{P^{1}}, 0,0\right)^{T} ; \\
\mathcal{Q}_{2} & =\left(0, P^{1}, P^{2}, P^{3}, \frac{\mathbf{Z}_{1}}{P^{1} P^{2} P^{3}}, 0,0,0\right)^{T},
\end{aligned}
$$

with $\mathbf{Z}_{1}>0 \Rightarrow \mathcal{I}_{4}\left(\mathcal{Q}_{2}\right)>0$, and $\mathcal{I}_{4}\left(\mathcal{Q}_{1}\right)>0$. In (A.12) $P^{1}, P^{2}, P^{3} \in \mathbb{R}_{0}^{+}$are the parameters of the three $S O(1,1)$ (generated by the three non-compact Cartan generators of $G_{4}=[S L(2, \mathbb{R})]^{3}$ ).

2. In the rank- $2 s t^{2}$ model $\left(G_{4}=[S L(2, \mathbb{R})]^{2}\right)$ there are 6 independent polynomial $G_{4}$-invariants depending on the $10 S O(1,1)$-invariant combinations. Thus, out of the 12 charges composing the charge vector $\mathbf{Q}^{\alpha}$ defined in (6.9), 4 charges can be set to zero by the action of the nondilatational 4 generators of $G_{4}$, spanning $\left[\frac{S L(2, \mathbb{R})}{S O(1,1)}\right]^{2}$ (namely, one conformal boost and one translational generator for each of the two $\frac{S L(2, \mathbb{R})}{S O(1,1)}$ factors). By recalling the definitions (A.3) of the $S O(1,1)$-invariant charge variables, a representative of a "minimal" charge configuration is e.g. given by

$$
\begin{aligned}
& \mathcal{Q}_{1}=\left(\frac{\mathbf{r}}{\mathbf{u} \mathbf{Z}_{\mathbf{1}}} P^{1}\left(P^{2}\right)^{2}, \frac{\mathbf{z}_{1}}{\mathbf{Z}_{1} \mathbf{u} \mathbf{u}_{\mathbf{2}}^{2}} P^{1}, \mathbf{u}_{\mathbf{2}} P^{2}, \frac{\mathbf{u} \mathbf{Z}_{\mathbf{1}}}{P^{1}\left(P^{2}\right)^{2}}, \frac{\mathbf{x} \mathbf{Z}_{1}}{\mathbf{z}_{1}} \frac{\mathbf{u} \mathbf{u}_{\mathbf{2}}^{2}}{P^{1}}, 0\right)^{T} ; \\
& \mathcal{Q}_{2}=\left(0, P^{1}, P^{2}, \frac{\mathbf{Z}_{1}}{P^{1}\left(P^{2}\right)^{2}}, 0,0\right)^{T},
\end{aligned}
$$

with $\mathbf{Z}_{1}>0 \Rightarrow \mathcal{I}_{4}\left(\mathcal{Q}_{2}\right)>0$, and $\mathcal{I}_{4}\left(\mathcal{Q}_{1}\right)>0$. In $\left(\right.$ A.13) $P^{1}, P^{2} \in \mathbb{R}_{0}^{+}$are the parameters of the two $S O(1,1)$ (generated by the two non-compact Cartan generators of $G_{4}=[S L(2, \mathbb{R})]^{2}$ ).

3. In the rank-1 irreducible $t^{3}$ model $\left(G_{4}=S L(2, \mathbb{R})\right)$ there are 5 independent polynomial $G_{4^{-}}$ invariants depending on the $7 S O(1,1)$-invariant combinations. Thus, out of the 8 charges composing the charge vector $\mathbf{Q}^{\alpha}$ defined in (7.15), 2 charges can be set to zero by the action of the non-dilatational 2 generators of $G_{4}$, spanning $\frac{S L(2, \mathbb{R})}{S O(1,1)}$ (namely, one conformal boost and one translational generator). By recalling the definitions (A.3) of the $S O(1,1)$-invariant charge 
variables, a representative of a "minimal" charge configuration is e.g. given by

$$
\begin{aligned}
& \mathcal{Q}_{1}=\left(\frac{\mathbf{r}}{\mathbf{u Z}_{\mathbf{1}}}(P)^{3}, \mathbf{u}_{\mathbf{2}} P, \frac{\mathbf{u} \mathbf{Z}_{\mathbf{1}}}{(P)^{3}}, 3 \frac{\mathbf{x}}{\mathbf{u}_{\mathbf{2}} P}\right)^{T} ; \\
& \mathcal{Q}_{2}=\left(0, P, \frac{\mathbf{Z}_{1}}{(P)^{3}}, 0\right)^{T},
\end{aligned}
$$

with $\mathbf{Z}_{1}>0 \Rightarrow \mathcal{I}_{4}\left(\mathcal{Q}_{2}\right)>0$, and $\mathcal{I}_{4}\left(\mathcal{Q}_{1}\right)>0$. In $($ A.14 $) P \in \mathbb{R}_{0}^{+}$is the parameter of the $S O(1,1)$ generated by the non-compact Cartan generator of $G_{4}=S L(2, \mathbb{R})$.

\section{B Constrained Calabi-Vesentini Basis for $t^{3}$ Model}

As mentioned in Sec. 7, the application of the Calabi-Vesentini [4, 44] T-tensor formalism to the $t^{3}$ model deserves a separate treatment, which we are going detail in the present Appendix.

In order to deal with this, let us start and recall some basic facts on the Calabi-Vesentini (CV) basis of $\mathcal{N}=2, d=4$ Jordan symmetric sequence. In particular, let us consider the stu model, whose CV basis has been explicitly discussed also in 41. Thus, for such a model, the symplectic sections are manifestly covariant under the whole $U$-duality group $S L(2, \mathbb{R}) \times S O(2,2) \sim[S L(2, \mathbb{R})]^{3}$ $(\Lambda=0,1,2,3)$

$$
\mathbf{V}\left(s, u_{1}, u_{2}\right) \equiv\left(\begin{array}{c}
X^{\Lambda} \\
F_{\Lambda}
\end{array}\right) \equiv\left(\begin{array}{c}
X^{\Lambda}\left(u_{1}, u_{2}\right) \\
s \eta_{\Lambda \Sigma} X^{\Sigma}\left(u_{1}, u_{2}\right)
\end{array}\right),
$$

where $X^{\Lambda}(u)$ satisfies the condition $X^{\Lambda}(u) \eta_{\Lambda \Sigma} X^{\Sigma}(u)=0$. The axion-dilaton field $s$ parameterizes the coset $\frac{S L(2, \mathbb{R})}{U(1)}$, whereas the two independent complex coordinates $u_{1}, u_{2}$ parameterize the coset $\frac{S O(2,2)}{S O(2) \times S O(2)}$. Note that, as shown in [44], in this symplectic frame a prepotential does not exist at all; however, it is still possible to calculate all the relevant geometrical quantities, using the standard formulæ of special Kähler geometry [44].

The relation between the CV symplectic frame specified by Eq. (B.1) and the "special coordinates" symplectic frame (whose manifest covariance is restricted to the $d=5 U$-duality group $[S O(1,1)]^{2}$; see e.g. the treatment in [41]) is given by [44, 41] ("SC" is acronym for "special coordinates")

$$
X_{C V}^{\Lambda}=\frac{1}{\sqrt{2}}\left(\begin{array}{c}
1-t u \\
-(t+u) \\
-(1+t u) \\
t-u
\end{array}\right) .
$$

Correspondingly, the $\mathrm{BH}$ charges in both symplectic frames are related by the following $S p(8, \mathbb{R})$ finite transformation [44, 41]

$$
\left(\begin{array}{c}
p^{\Lambda} \\
q_{\Lambda}
\end{array}\right)_{s t u, C V}=\frac{1}{\sqrt{2}}\left(\begin{array}{c}
p^{0}-q_{1} \\
-p^{2}-p^{3} \\
-p^{0}-q_{1} \\
p^{2}-p^{3} \\
p^{1}+q_{0} \\
-q_{2}-q_{3} \\
p^{1}-q_{0} \\
q_{2}-q_{3}
\end{array}\right)_{s t u, S C} .
$$

Starting from the CV basis of the stu model introduced above, the manifestly $(S L(2, \mathbb{R}) \times S O(2,1))$ covariant $\mathrm{CV}$ basis for the $s t^{2}$ model can be obtained by performing the following charge identifica- 
tions:

$$
\left\{\begin{array}{l}
p_{s t u, S C}^{2}=p_{s t u, S C}^{3} \equiv p_{s t^{2}, S C}^{2} \\
q_{2, s t u, S C}=q_{3, s t u, S C} \equiv \frac{1}{2} q_{2, s t u, S C} .
\end{array}\right.
$$

By so doing, the $S p(8, \mathbb{R})$ transformation (B.3) reduces to the following $S p(6, \mathbb{R})$ transformation $(\Lambda=0,1,2)$ :

$$
\left(\begin{array}{c}
p^{\Lambda} \\
q_{\Lambda}
\end{array}\right)_{s t^{2}, C V}=\frac{1}{\sqrt{2}}\left(\begin{array}{c}
p^{0}-q_{1} \\
-2 p^{2} \\
-p^{0}-q_{1} \\
p^{1}+q_{0} \\
-q_{2} \\
p^{1}-q_{0}
\end{array}\right)_{s t^{2}, S C} .
$$

On the other hand, if one wants to adapt the CV basis for stu model introduced above to the $t^{3}$ model, the following charge identifications are to be performed:

$$
\left\{\begin{array}{l}
p_{s t u, S C}^{1}=p_{s t u, S C}^{2}=p_{s t u, S C}^{3} \equiv p_{t^{3}, S C}^{1} \\
q_{1, s t u, S C}=q_{2, s t u, S C}=q_{3, s t u, S C} \equiv \frac{1}{3} q_{1, t^{3}, S C} .
\end{array}\right.
$$

Through these identifications, the $S p(8, \mathbb{R})$ transformation $(\overline{B .3})$ reduces to the following $S p(6, \mathbb{R})$ transformation $(\Lambda=0,1,2)$

$$
\left(\begin{array}{c}
p^{\Lambda} \\
q_{\Lambda}
\end{array}\right)_{t^{3}, C V}=\frac{1}{\sqrt{2}}\left(\begin{array}{c}
p^{0}-q_{1} / 3 \\
-2 p^{1} \\
-p^{0}-q_{1} / 3 \\
p^{1}+q_{0} \\
-2 q_{1} / 3 \\
p^{1}-q_{0}
\end{array}\right)_{t^{3}, S C} .
$$

Notice that still the symplectic index $\Lambda$ runs $0,1,2$, thus there would be six charges, not consistent with the four magnetic and electric charges of the $t^{3}$ model. In fact, the $p^{\Lambda}$ and $q_{\Lambda} 3$-vectors of (B.7) in the CV basis are not independent, but rather they are constrained by the two relations

$$
t^{3}, C V:\left\{\begin{array}{l}
p^{0}+p^{2}-q_{1}=0 \\
q_{0}+q_{2}+p^{1}=0
\end{array}\right.
$$

This yields to a consistent counting, because two real 3-vectors $p^{\Lambda}$ and $q_{\Lambda}$ with two real constraints (B.8) corresponds to four real charge degrees of freedom, namely the four charges of the $t^{3}$ model itself. Eqs. (B.7) and (B.8) defined a "constrained" CV symplectic frame for the $t^{3}$ model.

Interestingly, the relations (B.8) can be recast in a covariant fashion using a real form of the Pauli matrices

$$
\sigma_{1} \equiv\left(\begin{array}{cc}
0 & 1 \\
1 & 0
\end{array}\right), \quad \sigma_{2} \equiv\left(\begin{array}{cc}
1 & 0 \\
0 & -1
\end{array}\right), \quad \sigma_{3} \equiv\left(\begin{array}{cc}
0 & -1 \\
1 & 0
\end{array}\right)
$$

and 3 -vector of 2-component spinors

$$
r^{\Lambda}=\left(\begin{array}{c}
p^{\Lambda} \\
\eta^{\Lambda \Sigma} q_{\Sigma}
\end{array}\right) \equiv\left(\begin{array}{c}
\vec{r}^{1} \\
\vec{r}^{2}
\end{array}\right)
$$

\footnotetext{
${ }^{6}$ For a discussion of the "stu $\rightarrow s t^{2} \rightarrow t^{3}$ degeneration" in a different symplectic frame (relevant for applications to Quantum Information Theory [66]), see e.g. the discussion in Sec. 5 of 42 .
} 
where the definition of the $S O(2,1)$-vectors $\vec{s}^{\alpha}(\alpha=1,2)$ is done for later convenience. By means of $(\underline{B .9})$ and (B.10), the constraints (B.8) can be recast a: 7

$$
\vec{\sigma} \cdot \vec{r}=0 .
$$

On a group theoretical perspective, the constraints (B.8) or (B.11) denote the projection on the 4 ( $\operatorname{spin} s=3 / 2)$ irrepr. in the tensor product of the irreprs. 3 ( $\operatorname{spin} s=1)$ and $\mathbf{2}(\operatorname{spin} s=1 / 2)$ of the $d=4 U$-duality group $S L(2, \mathbb{R})$ of the $t^{3}$ model:

$$
\text { "st } t^{2} \rightarrow t^{3} \text { reduction": } \mathbf{3} \times \mathbf{2}=\begin{gathered}
\mathbf{4} \\
t^{3}
\end{gathered}+\underset{\mathbf{p r o j e c t e d ~ o u t ~ b y ~} \vec{\sigma} \cdot \vec{r}=0}{\mathbf{2}} .
$$

This is consistent with the above treatment, because the tensor product $\mathbf{3} \times \mathbf{2}$ realizes the "st $\rightarrow t^{2}$ reduction" of the charge repr. $(\mathbf{3}, \mathbf{2})$ of the $U$-duality $S L(2, \mathbb{R}) \times S O(2,1) \sim[S L(2, \mathbb{R})]^{2}$ of the $s t^{2}$ model down to the charge irrepr. 4 of the $U$-duality $S L(2, \mathbb{R})$ of the $t^{3}$ model itself.

In the case of an extremal BH 2-center solution, within this "constrained" CV symplectic frame, one can consider the $\mathbb{T}$-tensor formalism introduced above for the $t^{3}$, by simply considering the $s t^{2}$ model in CV basis and implementing the constraints (B.8) (or, equivalently, (B.11)).

The center 1 is constrained by (B.11), whereas the center 2 is constrained by

$$
\begin{aligned}
\vec{\sigma} \cdot \vec{s} & =0 ; \\
s^{\Lambda} & =\left(\begin{array}{c}
P^{\Lambda} \\
\eta^{\Lambda \Sigma} Q_{\Sigma}
\end{array}\right) \equiv\left(\begin{array}{c}
\vec{s}^{1} \\
\vec{s}^{2}
\end{array}\right) .
\end{aligned}
$$

A consequence of relations (B.11) and $(\underline{B .13})$ can be proved to be

$$
4(p \cdot P)(q \cdot Q)-4(p \cdot Q)(P \cdot q)-(\vec{p} \times \vec{Q}-\vec{q} \times \vec{P})^{2}=(p \cdot Q-P \cdot q)^{2},
$$

where " $\times$ " denotes the exterior product of the 3 -vectors $\vec{p} \equiv p^{\Lambda}, \vec{Q} \equiv Q^{\Lambda}$, etc., and the square in the last term in the l.h.s. is performed with the $S O(2,1)$-metric $\eta^{\Lambda \Sigma}$. Note that, by means of definitions (3.12) and (3.13), (B.15) is equivalent to the vanishing of the $S L_{h}(2, \mathbb{R})$-singlet $\mathcal{X}$ (see Eq. (7.16)).

In order to prove (B.15), we will work with complex groups. By recalling that Greek lowercase indices are spinor (e.g. $\alpha=1,2)$ - whereas Latin lowercase indices are vector (e.g. $i=1,2,3)$ - of $S L(2, \mathbb{C})$, one starts and introduces the spinor

$$
(\vec{\sigma} \cdot \vec{r})_{\alpha} \equiv \sigma_{\alpha \beta}^{i} r_{i}^{\beta}
$$

and the vector-spinor:

$$
(\vec{\sigma} \vec{\sigma} \cdot \vec{s})_{\alpha}^{i} \equiv \sigma_{\alpha \beta}^{i} \sigma_{\gamma}^{j \mid \beta} s_{j}^{\gamma}
$$

By expanding the products of $\sigma$-matrices through the Fierz identities, and elaborating the $\epsilon_{i j k} k^{\text {-symbols, }}$ one obtains that

$$
\begin{aligned}
(\vec{\sigma} \cdot \vec{r})_{\alpha}(\vec{\sigma} \vec{\sigma} \cdot \vec{s})_{\beta} \epsilon^{\alpha \beta=} & -4 i\left(\vec{r}^{\alpha} \times \vec{s}^{\beta}\right) \epsilon_{\alpha \beta}-4\left(\vec{r}^{\alpha} \cdot \vec{s}^{\beta}\right) \vec{\sigma}_{\alpha \beta} \\
& +4\left(\vec{\sigma}_{\alpha \beta} \cdot \vec{r}^{\alpha}\right) \vec{s}^{\beta}-2\left(\vec{\sigma}_{\alpha \beta} \cdot \vec{s}^{\alpha}\right) \vec{r}^{\beta},
\end{aligned}
$$

\footnotetext{
${ }^{7}$ Note that the quartic invariant polynomial of the 4 (spin $\left.s=3 / 2\right)$ irrepr. of $S L(2, \mathbb{R})$ can be written as 67

$$
\mathcal{I}_{4}=\left|\vec{r}^{1} \times \vec{r}^{2}\right|^{2}=\left|\vec{r}^{1}\right|^{2}\left|\vec{r}^{2}\right|^{2}-\left(\vec{r}^{1} \cdot \vec{r}^{2}\right)^{2},
$$

constrained by (B.11), where " $\times$ " denotes the exterior product of the 3 -vectors $\vec{r}^{1}$ and $\vec{r}^{2}$, and the square norms are computed with the $S O(2,1)$-metric $\eta^{\Lambda \Sigma}$. In the "special coordinates" symplectic frame used in Sect. $7 \mathcal{I}_{4}$ is given by Eq. (7.6).
} 
which, under the constraints (B.11) and (B.13), yields

$$
i\left(\vec{r}^{\alpha} \times \vec{s}^{\beta}\right) \epsilon_{\alpha \beta}=-\left(\vec{r}^{\alpha} \cdot \vec{s}^{\beta}\right) \vec{\sigma}_{\alpha \beta} .
$$

By squaring (B.19) and using the Fierz identities, the following result is finally achieved:

$$
\begin{gathered}
-\left(\vec{r}_{1} \times \vec{s}_{2}-\vec{r}_{2} \times \vec{s}_{1}\right)^{2}=\left(\vec{r}^{\alpha} \cdot \vec{s}^{\beta}\right)\left(\vec{r}^{\gamma} \cdot \vec{s}^{\delta}\right) \vec{\sigma}_{\alpha \beta} \cdot \vec{\sigma}_{\gamma \delta} \\
=\left(\vec{r}_{1} \cdot \vec{s}_{2}-\vec{r}_{2} \cdot \vec{s}_{1}\right)^{2}-4\left(\vec{r}_{1} \times \vec{r}_{2}\right) \cdot\left(\vec{s}_{1} \times \vec{s}_{2}\right) \\
\text { 步 } \\
4\left(\vec{r}_{1} \times \vec{r}_{2}\right) \cdot\left(\vec{s}_{1} \times \vec{s}_{2}\right)-\left(\vec{r}_{1} \times \vec{s}_{2}-\vec{r}_{2} \times \vec{s}_{1}\right)^{2}=\left(\vec{r}_{1} \cdot \vec{s}_{2}-\vec{r}_{2} \cdot \vec{s}_{1}\right)^{2},
\end{gathered}
$$

which, through definitions (B.10) and (B.14), matches Eq. (B.15).

\section{A Complete Basis for $S O(n, \mathbb{C})$-invariant Polynomials}

We now proceed to prove that every $S O(n, \mathbb{C})$-invariant polynomial $\mathcal{P}(\mathbf{A})$ of a $n \times n$ complex symmetric matrix $\mathbf{A}=\mathbf{A}^{T} \in M_{n}(\mathbb{C})$ is a polynomial of $\left\{\operatorname{Tr}\left(\mathbf{A}^{p}\right)\right\}_{1 \leqslant p \leqslant n}$ :

$$
\forall g \in S O(n, \mathbb{C}), \mathcal{P}\left(g^{-1} \mathbf{A} g\right)=\mathcal{P}(\mathbf{A}) \Longrightarrow \mathcal{P}(\mathbf{A})=\mathcal{Q}\left(\operatorname{Tr} \mathbf{A}, \operatorname{Tr}\left(\mathbf{A}^{2}\right), \ldots, \operatorname{Tr}\left(\mathbf{A}^{n}\right)\right),
$$

where $\mathcal{Q}$ denotes some polynomial.

In order to prove (C.1), we start by observing that $\mathcal{P}\left(g^{-1} \mathbf{A} g\right)$ (which is holomorphic in $g$ and $\mathbf{A}$ ) is determined by analytic continuation 9 from its value taken for $g \in S O(n, \mathbb{R})$ and for $\mathbf{A}=\mathbf{A}^{T} \in$ $G L(n, \mathbb{R})$ (e.g. through a convergent series in the neighbourhood of any real point in $\mathbb{C}$ ).

By virtue of this observation, it then suffices to prove (C.1) for $g \in S O(n, \mathbb{R})$ and for $\mathbf{A}=\mathbf{A}^{T} \in$ $M_{n}(\mathbb{R})$.

In order to do so, we notice that $\mathbf{A}$ real symmetric can always be diagonalised through a suitable transformation $t \in S O(n, \mathbb{R})$, yielding real eigenvalues $\left\{\lambda_{1}, \ldots, \lambda_{n}\right\}$. Thus, every $S O(n, \mathbb{R})$-invariant polynomial $\mathcal{P}(\mathbf{A})$ of a real symmetric $n \times n$ matrix $\mathbf{A}$ is also a polynomial in $\left\{\lambda_{1}, \ldots, \lambda_{n}\right\}$; its $S O(n, \mathbb{R})$ invariance implie 10 that it is symmetric under even permutations of the indices $\{1, \ldots, n\}$. Furthermore, such a polynomial in $\left\{\lambda_{1}, \ldots, \lambda_{n}\right\}$ can be split into a symmetric component and into an antisymmetric component under odd permutations of the indices $\{1, \ldots, n\}$ :

- the symmetric component is given by $\mathfrak{P}\left(\lambda_{1}, \ldots, \lambda_{n}\right)$, a polynomial which is symmetric under all permutations of indices $\left\{\lambda_{1}, \ldots, \lambda_{n}\right\}$. Its functional dependence on the eigenvalues $\left\{\lambda_{1}, \ldots, \lambda_{n}\right\}$ can be proved to be as follows (see e.g. [68]):

$$
\mathfrak{P}\left(\lambda_{1}, \ldots, \lambda_{n}\right)=\widetilde{\mathfrak{P}}\left(\sigma_{p} \equiv \sum_{i} \lambda_{i}^{p}, 1 \leqslant p \leqslant n\right)=\mathcal{Q}\left(\operatorname{Tr} \mathbf{A}, \operatorname{Tr}\left(\mathbf{A}^{2}\right), \ldots, \operatorname{Tr}\left(\mathbf{A}^{n}\right)\right) .
$$

- the anti-symmetric component is of the form (recall ([C.2); see e.g. 68])

$$
\mathfrak{P}^{\prime}\left(\lambda_{1}, \ldots, \lambda_{n}\right) \Delta\left(\lambda_{1}, \ldots, \lambda_{n}\right)=\mathcal{Q}^{\prime}\left(\operatorname{Tr} \mathbf{A}, \operatorname{Tr}\left(\mathbf{A}^{2}\right), \ldots, \operatorname{Tr}\left(\mathbf{A}^{n}\right)\right) \Delta\left(\lambda_{1}, \ldots, \lambda_{n}\right),
$$

where

$$
\Delta\left(\lambda_{1}, \ldots, \lambda_{n}\right) \equiv \prod_{1 \leqslant i<j \leqslant n}\left(\lambda_{j}-\lambda_{i}\right)=\operatorname{det}\left(\begin{array}{cccc}
1 & \lambda_{1} & \ldots & \lambda_{1}^{n-1} \\
1 & \lambda_{2} & \ldots & \lambda_{2}^{n-1} \\
\ldots & \ldots & \ldots & \ldots \\
1 & \lambda_{n} & \ldots & \lambda_{n}^{n-1}
\end{array}\right)
$$

\footnotetext{
${ }^{8}$ For $p>n, \operatorname{Tr}\left(\mathbf{A}^{p}\right)$ can be expressed in terms of $\left\{\operatorname{Tr}\left(\mathbf{A}^{p}\right)\right\}_{1 \leqslant p \leqslant n}$ since, by virtue of the Cayley-Hamilton Theorem, A fulfills its characteristic equation $\operatorname{det}\left(\lambda \mathbb{I}_{n}-\mathbf{A}\right)=0\left(\mathbb{I}_{n}\right.$ denoting the $n \times n$ identity).

${ }^{9}$ We thank Prof. Michel Dubois Violette for this argument.

${ }^{10}$ Indeed, an even permutation of the indices $\{1, \ldots, n\}$ can be represented by a suitable transformation of $S O(n, \mathbb{R})$.
} 
is the Vandermonde determinant. It should be remarked that $\Delta$ is not a polynomial in $\mathbf{A}$, because it is a square root of the discriminant of the characteristic polynomial $\operatorname{det}\left(\lambda \mathbb{I}_{n}-\mathbf{A}\right)$. Indeed, in general the transformation $t \in S O(n, \mathbb{R})$ which diagonalizes $\mathbf{A}$ is not a polynomial in $\mathbf{A}$ itself, because, due to orthonormalisation of the eigenvectors of $\mathbf{A}$, it involves square roots.

Therefore, since we restrict to consider $S O(n, \mathbb{R})$-invariant polynomials in the real symmetric matrix A, the observations above lead to the conclusion that such polynomials necessarily are of the form (C.2). The analytic continuation of this result to $\mathbb{C}$ yields the proof of (C.1).

Thus, as mentioned in Sec. 8, by means of Newton's formula [61 (cfr. Eqs. (8.7)-(8.10) in the case $n=4)$, the characteristic polynomial $\operatorname{det}\left(\lambda \mathbb{I}_{n}-\mathbf{A}\right)$ can be considered as a generating function for the ring of $S O(n, \mathbb{C})$-invariant polynomials of $\mathbf{A}=\mathbf{A}^{T} \in M_{n}(\mathbb{C})$.

\section{References}

[1] S. Ferrara, R. Kallosh and A. Strominger, $\mathcal{N}=2$ extremal black holes, Phys. Rev. D52, 5412 (1995), hep-th/9508072. A. Strominger, Macroscopic entropy of $\mathcal{N}=2$ extremal black holes, Phys. Lett. B383, 39 (1996), hep-th/9602111. S. Ferrara and R. Kallosh, Supersymmetry and attractors, Phys. Rev. D54, 1514 (1996), hep-th/9602136. S. Ferrara and R. Kallosh, Universality of supersymmetric attractors, Phys. Rev. D54, 1525 (1996), hep-th/9603090.

[2] S. Ferrara, G. W. Gibbons and R. Kallosh, Black Holes and Critical Points in Moduli Space, Nucl. Phys. B500, 75 (1997), hep-th/9702103.

[3] F. Denef, Supergravity flows and D-brane stability, JHEP 0008, 050 (2000), hep-th/0005049.

[4] F. Denef, B. R. Greene and M. Raugas, Split attractor flows and the spectrum of BPS D-branes on the quintic, JHEP 0105, 012 (2001), hep-th/0101135.

[5] B. Bates and F. Denef, Exact solutions for supersymmetric stationary black hole composites, arXiv:hep-th/0304094.

[6] R. Kallosh, N. Sivanandam, and M. Soroush, Exact Attractive Non-BPS STU Black Holes, Phys. Rev. D74, 065008 (2006), hep-th/0606263.

[7] F. Denef and G. W. Moore, Split States, Entropy Enigmas, Holes and Halos, hep-th/0702146. F. Denef, D. Gaiotto, A. Strominger, D. Van den Bleeken and X. Yin, Black Hole Deconstruction, hep-th/0703252. F. Denef and G. W. Moore, How many black holes fit on the head of a pin?, Gen. Rel. Grav. 39, 1539 (2007), arXiv:0705.2564 [hep-th].

[8] D. Gaiotto, W. W. Li and M. Padi, Non-Supersymmetric Attractor Flow in Symmetric Spaces, JHEP 0712, 093 (2007), arXiv:0710.1638 [hep-th].

[9] M. C. N. Cheng and E. P. Verlinde, Wall Crossing, Discrete Attractor Flow, and Borcherds Algebra, SIGMA 4, 068 (2008), arXiv:0806.2337 [hep-th].

[10] E. G. Gimon, F. Larsen and J. Simon, Constituent Model of Extremal non-BPS Black Holes, JHEP 0907, 052 (2009), arXiv:0903.0719 [hep-th].

[11] A. Castro and J. Simon, Deconstructing the D0-D6 system, JHEP 0905, 078 (2009), arXiv:0903.5523 [hep-th].

[12] J. R. David, On walls of marginal stability in $\mathcal{N}=2$ string theories, JHEP 0908, 054 (2009), arXiv:0905.4115 [hep-th]. 
[13] J. Manschot, Stability and Duality in $\mathcal{N}=2$ Supergravity, Commun. Math. Phys. 299, 651 (2010), arXiv:0906.1767 [hep-th].

[14] P. Galli and J. Perz, Non-supersymmetric extremal multicenter black holes with superpotentials, JHEP 1002, 102 (2010), arXiv:0909.5185 [hep-th].

[15] E. Andriyash, F. Denef, D. L. Jafferis and G. W. Moore, Wall-crossing from supersymmetric galaxies, arXiv:1008.0030 [hep-th]. E. Andriyash, F. Denef, D. L. Jafferis and G. W. Moore, Bound state transformation walls, arXiv:1008.3555 [hep-th].

[16] A. Sen, Walls of Marginal Stability and Dyon Spectrum in $\mathcal{N}=4$ Supersymmetric String Theories, JHEP 0705, 039 (2007), hep-th/0702141. A. Sen, Two Centered Black Holes and $\mathcal{N}=4$ Dyon Spectrum, JHEP 0709, 045 (2007), arXiv:0705.3874 [hep-th]. A. Sen, $\mathcal{N}=8$ Dyon Partition Function and Walls of Marginal Stability, JHEP 0807, 118 (2008), arXiv:0803.1014 [hep-th]. A. Sen, Wall Crossing Formula for $\mathcal{N}=4$ Dyons: A Macroscopic Derivation, JHEP 0807, 078 (2008), arXiv:0803.3857.

[17] J. Manschot, B. Pioline and A. Sen, Wall Crossing from Boltzmann Black Holes, arXiv: 1011.1258 [hep-th].

[18] J. Rahmfeld, Extremal black holes as bound states, Phys. Lett. B372, 198 (1996), hep-th/9512089; M. J. Duff and J. Rahmfeld, Bound states of black holes and other p-branes, Nucl. Phys. B481, 332 (1996), hep-th/9605085.

[19] C. M. Hull and P. K. Townsend, Unity of Superstring Dualities, Nucl. Phys. B438, hep-th/9410167.

[20] S. Ferrara and M. Günaydin, Orbits of Exceptional Groups, Duality and BPS States in String Theory, Int. J. Mod. Phys. A13, 2075 (1998), hep-th/9708025.

[21] H. Lu, C. N. Pope and K. S. Stelle, Multiplet Structures of BPS Solitons, Class. Quant. Grav. 15, 537 (1998), hep-th/9708109.

[22] R. D'Auria, S. Ferrara, M. A. Lledó, On central charges and Hamiltonians for 0-brane dynamics, Phys. Rev. D60, 084007 (1999), hep-th/9903089.

[23] S. Bellucci, S. Ferrara, M. Günaydin and A. Marrani, Charge orbits of symmetric special geometries and attractors, Int. J. Mod. Phys. A21, 5043 (2006), hep-th/0606209.

[24] L. Andrianopoli, R. D'Auria, S. Ferrara and M. Trigiante, Extremal black holes in supergravity, Lect. Notes Phys. 737, 661 (2008), hep-th/0611345.

[25] S. Bellucci, S. Ferrara, R. Kallosh and A. Marrani, Extremal Black Hole and Flux Vacua Attractors, Lect. Notes Phys. 755, 115 (2008), arXiv:0711.4547 [hep-th].

[26] G. Bossard, H. Nicolai and K. S. Stelle, Universal BPS Structure of Stationary Supergravity Solutions, JHEP 0907, 003 (2009), arXiv:0902.4438 [hep-th].

[27] B. L. Cerchiai, S. Ferrara, A. Marrani and B. Zumino, Duality, Entropy and ADM Mass in Supergravity, Phys. Rev. D79, 125010 (2009), arXiv:0902.3973 [hep-th].

[28] S. Bellucci, S. Ferrara, M. Günaydin and A. Marrani, SAM Lectures on Extremal Black Holes in $d=4$ Extended Supergravity, arXiv:0905.3739 [hep-th].

[29] L. Andrianopoli, R. D'Auria and S. Ferrara, U-Invariants, Black-Hole Entropy and Fixed Scalars, Phys. Lett. B403, 12 (1997), hep-th/9703156. 
[30] S. Ferrara and A. Marrani, $\mathcal{N}=8$ non-BPS Attractors, Fixed Scalars and Magic Supergravities, Nucl. Phys. B788, 63 (2008), arXiv:0705.3866 [hep-th]. S. Ferrara and A. Marrani, On the Moduli Space of non-BPS Attractors for $\mathcal{N}=2$ Symmetric Manifolds, Phys. Lett. B652, 111 (2007), arXiv:0706.1667 [hep-th]. S. Bellucci, S. Ferrara, R. Kallosh and A. Marrani, Extremal Black Hole and Flux Vacua Attractors, Lect. Notes Phys. 755, 115 (2008), arXiv:0711.4547 [hep-th].

[31] J. F. Luciani, Coupling of O(2) Supergravity with Several Vector Multiplets, Nucl. Phys. B132, 325 (1978).

[32] L. Castellani, A. Ceresole, S. Ferrara, R. D'Auria, P. Fré and E. Maina, The Complete $\mathcal{N}=3$ Matter Coupled Supergravity, Nucl. Phys. B268, 317 (1986).

[33] E. Cremmer, J. Scherk and S. Ferrara, SU(4) Invariant Supergravity Theory, Phys. Lett. B74, 61 (1978).

[34] B. de Wit, F. Vanderseypen and A. Van Proeyen, Symmetry Structures of Special Geometries, Nucl. Phys. B400, 463 (1993), hep-th/9210068.

[35] M. Günaydin, G. Sierra and P. K. Townsend, Exceptional Supergravity Theories and the Magic Square, Phys. Lett. B133, 72 (1983).

[36] M. Günaydin, G. Sierra and P. K. Townsend, The Geometry of $N=2$ Maxwell-Einstein Supergravity and Jordan Algebras, Nucl. Phys. B242, 244 (1984).

[37] S. Ferrara, A. Marrani and E. Orazi, Split Attractor Flow in $\mathcal{N}=2$ Minimally Coupled Supergravity, arXiv:1010.2280 [hep-th].

[38] M. Günaydin, Lectures on Spectrum Generating Symmetries and U-Duality in Supergravity, Extremal Black Holes, Quantum Attractors and Harmonic Superspace, arXiv:0908.0374 [hep-th].

[39] S. Ferrara and A. Marrani, Matrix Norms, BPS Bounds and Marginal Stability in $\mathcal{N}=8$ Supergravity, arXiv:1009.3251 [hep-th].

[40] M. J. Duff, J. T. Liu and J. Rahmfeld, Four-dimensional string/string/string triality, Nucl. Phys. B459, 125 (1996), hep-th/9508094.

[41] K. Behrndt, R. Kallosh, J. Rahmfeld, M. Shmakova and W. K. Wong, STU black holes and string triality, Phys. Rev. D54, 6293 (1996), hep-th/9608059.

[42] S. Bellucci, A. Marrani, E. Orazi and A. Shcherbakov, Attractors with Vanishing Central Charge, Phys. Lett. B655, 185 (2007), arXiv:0707.2730.

[43] E. Calabi and E. Vesentini, On Compact, Locally Symmetric Kähler Manifolds, Ann. Math. 71, $472(1960)$.

[44] A. Ceresole, R. D'Auria, S. Ferrara and A. Van Proeyen, Duality Transformations in Supersymmetric Yang-Mills Theories coupled to Supergravity, Nucl. Phys. B444, 92 (1995), hep-th/9502072.

[45] A. Ceresole, R. D'Auria and S. Ferrara, The Symplectic structure of $\mathcal{N}=2$ supergravity and its central extension, Nucl. Phys. Proc. Suppl. 46, 67 (1996), hep-th/9509160.

[46] S. Bellucci, S. Ferrara, A. Marrani and A. Yeranyan, stu Black Holes Unveiled, Entropy 10 (4), 507 (2008), arXiv:0807.3503. 
[47] A. Ceresole, G. Dall'Agata, S. Ferrara and A. Yeranyan, Universality of the Superpotential for $d=4$ Extremal Black Holes, arXiv:0910.2697 [hep-th].

[48] S. Cecotti, S. Ferrara and L. Girardello, Geometry of Type II Superstrings and the Moduli of Superconformal Field Theories, Int. J. Mod. Phys. A4, 2475 (1989).

[49] M. Shmakova, Calabi-Yau black holes, Phys. Rev. D56, 540 (1997), hep-th/9612076.

[50] A. Cayley, On the Theory of Linear Transformations, Camb. Math. J. 4, 193 (1845).

[51] S. Ferrara, E. G. Gimon and R. Kallosh, Magic supergravities, $\mathcal{N}=8$ and black hole composites, Phys. Rev. D74, 125018 (2006), hep-th/0606211.

[52] E. Cremmer and A. Van Proeyen, Classification of Kähler Manifolds in $\mathcal{N}=2$ Vector Multiplet Supergravity Couplings, Class. Quant. Grav. 2, 445 (1985).

[53] S. Ferrara and J. M. Maldacena, Branes, central charges and U duality invariant BPS conditions, Class. Quant. Grav. 15, 749 (1998), hep-th/9706097.

[54] L. Andrianopoli, R. D'Auria, S. Ferrara and M. Trigiante, Fake Superpotential for Large and Small Extremal Black Holes, JHEP 1008, 126 (2010), arXiv:1002.4340 [hep-th].

[55] M. Cvetic and D. Youm, Dyonic BPS saturated black holes of heterotic string on a six torus, Phys. Rev. D53, 584 (1996), hep-th/9507090.

[56] M. Cvetic and A. A. Tseytlin, General class of BPS saturated dyonic black holes as exact superstring solutions, Phys. Lett. B366, 95 (1996), hep-th/9510097. M. Cvetic and A. A. Tseytlin, Solitonic strings and BPS saturated dyonic black holes, Phys. Rev. D53, 5619 (1996); Erratumibid. D55, 3907 (1997), hep-th/9512031.

[57] K. Saraikin and C. Vafa, Non-supersymmetric black holes and topological strings, Class. Quant. Grav. 25, 095007 (2008), hep-th/0703214.

[58] P. K. Tripathy and S. P. Trivedi, Non-supersymmetric attractors in string theory, JHEP 0603, 022 (2006), hep-th/0511117.

[59] A. Ceresole, G. Dall'Agata, S. Ferrara and A. Yeranyan, First Order Flows for $\mathcal{N}=2$ Extremal Black Holes and Duality Invariants, Nucl. Phys. B824, 239 (2010), arXiv:0908.1110 [hep-th].

[60] See e.g. : F. R. Gantmacher, "Matrix Theory, Vol. 1", Chelsea Publishing Company (New York, 1959).

[61] B. Sturmfels, "Algorithms in Invariant Theory", (New York, Wiley, 1992).

[62] E. Bergshoeff, I. G. Koh and E. Sezgin, Coupling of Yang-Mills to $\mathcal{N}=4, D=4$ Supergravity, Phys. Lett. B155, 71 (1985).

[63] M. de Roo and P. Wagemans, Gauge Matter Coupling In $\mathcal{N}=4$ Supergravity, Nucl. Phys. B262, 644 (1985).

[64] S. Ferrara, A. Gnecchi and A. Marrani, $d=4$ Attractors, Effective Horizon Radius and Fake Supergravity, Phys. Rev. D78, 065003 (2008), arXiv:0806.3196 [hep-th].

[65] Invited Talk by S. Ferrara at the "25 th Anniversary of Dirac Medal" Conference, Nov. 10 (2010), ICTP, Trieste, Italy. 
[66] See e.g. the following papers (and Refs. therein) : M. J. Duff, String triality, black hole entropy and Cayley's hyperdeterminant, Phys. Rev. D76, 025017 (2007), hep-th/0601134. R. Kallosh and A. Linde, Strings, black holes, and quantum information, Phys. Rev. D73, 104033 (2006), hep-th/0602061. M. J. Duff and S. Ferrara, Black hole entropy and quantum information, Lect. Notes Phys. 755, 93 (2008), arXiv:hepth/0612036. L. Borsten, D. Dahanayake, M. J. Duff, H. Ebrahim and W. Rubens, Black Holes, Qubits and Octonions, Phys. Rept. 471, 113 (2009), arXiv:0809.4685 [hep-th]. P. Levay, STU Black Holes as Four Qubit Systems, Phys. Rev. D82, 026003 (2010), arXiv: 1004.3639 [hep-th]. L. Borsten, D. Dahanayake, M. J. Duff, A. Marrani and W. Rubens, Four-Qubit Entanglement from String Theory, Phys. Rev. Lett. 105, 100507 (2010), arXiv: 1005.4915 [hep-th].

[67] C. Procesi, "Lie Groups : An Approach through Invariants and Representations" Universitext, Springer Verlag (Berlin, 2006).

[68] I. G. McDonald, "Symmetric Functions and Hall Polynomials", Oxford Mathematical Monographs, Clarendon Press (Oxford, 1979). 